\title{
Invariant Dirac Operators, Harmonic Spinors, and Vanishing Theorems in CR Geometry
}

\author{
Felipe LEITNER
}

Universität Greifswald, Institut für Mathematik und Informatik, Walter-Rathenau-Str. 47, D-17489 Greifswald, Germany

E-mail: felipe.leitner@uni-greifswald.de

Received July 23, 2020, in final form January 22, 2021; Published online February 04, 2021

https://doi.org/10.3842/SIGMA.2021.011

\begin{abstract}
We study Kohn-Dirac operators $D_{\theta}$ on strictly pseudoconvex CR manifolds with $\operatorname{spin}^{\mathbb{C}}$ structure of weight $\ell \in \mathbb{Z}$. Certain components of $D_{\theta}$ are CR invariants. We also derive $\mathrm{CR}$ invariant twistor operators of weight $\ell$. Harmonic spinors correspond to cohomology classes of some twisted Kohn-Rossi complex. Applying a Schrödinger-Lichnerowicz-type formula, we prove vanishing theorems for harmonic spinors and (twisted) Kohn-Rossi groups. We also derive obstructions to positive Webster curvature.
\end{abstract}

Key words: CR geometry; spin geometry; Kohn-Dirac operator; harmonic spinors; KohnRossi cohomology; vanishing theorems

2020 Mathematics Subject Classification: 32V05; 53C27; 58J50; 32L20

\section{Introduction}

The classical Schrödinger-Lichnerowicz formula

$$
D^{2}=\Delta+\frac{\text { scal }}{4}
$$

of Riemannian geometry relates the square of the Dirac operator to the spinor Laplacian and scalar curvature. This Weitzenböck formula can be used to prove vanishing theorems for harmonic spinors on closed manifolds. Via Hodge theory and Dolbeault's theorem this give rise to vanishing theorems for holomorphic cohomology on Kähler manifolds (see [5]). Moreover, via the index theorem for elliptic differential operators, the $\hat{\mathcal{A}}$-genus is understood to be an obstruction to positive scalar curvature on spin manifolds (see [14]).

Due to J.J. Kohn there is also an harmonic theory for the Kohn-Laplacian on strictly pseudoconvex CR manifolds (see [4, 7]). Even though the Kohn-Laplacian is not elliptic, this theory shows that classes in the cohomology groups of the tangential Cauchy-Riemann complex (or Kohn-Rossi complex) are represented by harmonic forms. In particular, the (non-extremal) Kohn-Rossi groups are finite dimensional over closed manifolds.

In [21] Tanaka describes this harmonic theory for the Kohn-Laplacian on $(p, q)$-forms with values in some $\mathrm{CR}$ vector bundle $E$ over (abstract) strictly pseudoconvex $\mathrm{CR}$ manifolds. The Kohn-Laplacian is defined with respect to some pseudo-Hermitian structure $\theta$ and the corresponding canonical connection. In particular, Tanaka derives Weitzenböck formulas and proves vanishing theorems for the Kohn-Rossi groups. On the other hand, in [18] R. Petit introduces spinor calculus and Dirac-type operators to strictly pseudoconvex CR manifolds with adapted pseudo-Hermitian structure (cf. also [12, 20]). Deriving some Schrödinger-Lichnerowicz-type formula for the Kohn-Dirac operator, this approach gives rise to vanishing theorems for harmonic spinors over closed CR manifolds (cf. also [9]). 
We study in this paper the Kohn-Dirac operator $D_{\theta}$ for $\operatorname{spin}^{\mathbb{C}}$ structures of weight $\ell \in \mathbb{Z}$ on strictly pseudoconvex CR manifolds with adapted pseudo-Hermitian structure $\theta$. Our construction of $D_{\theta}$ uses the Webster-Tanaka spinor derivative, only. The Kohn-Dirac operator $D_{\theta}$ does not behave naturally with respect to conformal changes of the underlying pseudo-Hermitian structure. However, similar as in Kähler geometry, the spinor bundle $\Sigma$ decomposes with respect to the CR structure into eigenbundles $\Sigma^{\mu_{q}}$ of certain eigenvalues $\mu_{q}$. For $\mu_{q}=-\ell$ the restriction $\mathcal{D}_{\ell}$ of the Kohn-Dirac operator to $\Gamma\left(\Sigma^{\mu_{q}}\right)$ acts CR-covariantly. This observation gives rise to CR invariants for the underlying strictly pseudoconvex CR manifold.

Complementary to $D_{\theta}$ we also have twistor operators. In the spin case [12] we discuss special solutions of the corresponding twistor equation, which realize some lower bound for the square of the first non-zero eigenvalue of the Kohn-Dirac operator $D_{\theta}$. For $\mu_{q}=\ell$ the corresponding twistor operator $\mathcal{P}_{\ell}$ is a $\mathrm{CR}$ invariant.

Analyzing the Clifford multiplication on the spinor bundle for $\operatorname{spin}^{\mathbb{C}}$ structures over strictly pseudoconvex CR manifolds shows that the Kohn-Dirac operator is a square root of the KohnLaplacian acting on $(0, q)$-forms with values in some CR line bundle $E$. Thus, our discussion of the Kohn-Dirac operator fits well to Kohn's harmonic theory, as described in [21]. In particular, harmonic spinors correspond to cohomology classes of certain twisted Kohn-Rossi complexes. Computing the curvature term of the corresponding Schrödinger-Lichnerowicz-type formula gives rise to vanishing theorems for twisted Kohn-Rossi groups.

For example, on a closed, strictly pseudoconvex CR manifold $M$ of even CR dimension $m \geq 2$ with spin structure given by a square root $\sqrt{\mathcal{K}}$ of the canonical line bundle, we have for $\mu_{q}=\ell=0$ the Schrödinger-Lichnerowicz-type formula

$$
\mathcal{D}_{0}^{*} \mathcal{D}_{0}=\Delta^{\mathrm{tr}}+\frac{\mathrm{scal}^{\mathrm{W}}}{4}
$$

for the CR-covariant component $\mathcal{D}_{0}$ of $D_{\theta}$, where $\Delta^{\text {tr }}$ denotes the spinor sub-Laplacian and scal ${ }^{\mathrm{W}}$ is the Webster scalar curvature. In this case harmonic spinors correspond to cohomology classes in the Kohn-Rossi group $H^{\frac{m}{2}}(M, \sqrt{\mathcal{K}})$. Positive Webster scalar curvature scal ${ }^{\mathrm{W}}>0$ on $M$ immediately implies that this Kohn-Rossi group is trivial. On the other hand, $H^{\frac{m}{2}}(M, \sqrt{\mathcal{K}}) \neq\{0\}$ poses an obstruction to the existence of any adapted pseudo-Hermitian structure $\theta$ on $M$ of positive Webster scalar curvature. In this case the Yamabe invariant in [6] for the given CR structure is non-positive.

In Sections 2 to 5 we introduce CR manifolds and pseudo-Hermitian geometry with spin ${ }^{\mathbb{C}}$ structures. In Section 6 the Kohn-Dirac and twistor operators are constructed. The CRcovariant components $\mathcal{D}_{\ell}$ and $\mathcal{P}_{\ell}$ are determined in Section 7. In Section 8 we recall the Schrödinger-Lichnerowicz-type formula and derive a basic vanishing theorem for harmonic spinors (see Proposition 8.1). Section 9 briefly reviews the harmonic theory for the Kohn-Laplacian. In Section 10 we derive vanishing theorems for twisted Kohn-Rossi groups. In Section 11 we discuss CR circle bundles of Kähler manifolds and relate holomorphic cohomology groups to Kohn-Rossi groups. Finally, in Section 12 we construct closed, strictly pseudoconvex CR manifolds with $H^{\frac{m}{2}}(M, \sqrt{\mathcal{K}}) \neq 0$.

\section{Strictly pseudoconvex CR structures}

Let $M^{n}$ be a connected and orientable, real $C^{\infty}$-manifold of odd dimension $n=2 m+1 \geq 3$, equipped with a pair $(H(M), J)$ of a corank 1 subbundle $H(M)$ of the tangent bundle $T(M)$ and a bundle endomorphism $J: H(M) \rightarrow H(M)$ with $J^{2}(X)=-X$ for any $X \in H(M)$. The 
Lie bracket $[\cdot, \cdot]$ of vector fields defines a bilinear skew pairing

$$
\begin{aligned}
\{\cdot, \cdot\}: H(M) \times H(M) & \rightarrow T(M) / H(M), \\
(X, Y) & \mapsto-[X, Y] \bmod H(M),
\end{aligned}
$$

with values in the real line bundle $T(M) / H(M)$.

We call the pair $(H(M), J)$ a strictly pseudoconvex $C R$ structure on $M$ (of hypersurface type and $\mathrm{CR}$ dimension $m \geq 1$ ) if the following conditions are satisfied:

- $\{J X, Y\}+\{X, J Y\}=0 \bmod H(M)$ for any $X, Y \in H(M)$ and

- the symmetric pairing $\{\cdot, J \cdot\}$ on $H(M)$ is definite, i.e., $\{X, J X\} \neq 0$ for any $X \neq 0$,

- the Nijenhuis tensor $N_{J}(X, Y)=[X, Y]-[J X, J Y]+J([J X, Y]+[X, J Y])$ vanishes identically for any $X, Y \in H(M)$.

Throughout this paper we will deal with strictly pseudoconvex CR structures on $M$. For example, in the generic case when the Levi form is non-degenerate, the smooth boundary of a domain of holomorphy in $\mathbb{C}^{m+1}$ is strictly pseudoconvex.

The complex structure $J$ extends $\mathbb{C}$-linearly to $H(M) \otimes \mathbb{C}$, the complexification of the Levi distribution, and induces a decomposition

$$
H(M) \otimes \mathbb{C}=T_{10} \oplus T_{01}
$$

into $\pm \mathrm{i}$-eigenbundles. Then a complex-valued $p$-form $\eta$ on $M$ is said to be of type $(p, 0)$ if $\iota_{Z} \eta=0$ for all $Z \in T_{01}$. This gives rise to the complex vector bundle $\Lambda^{p, 0}(M)$ of $(p, 0)$-forms on $M$. For the $(m+1)$ st exterior power $\Lambda^{m+1,0}(M)$ of $\Lambda^{1,0}(M)$ we write $\mathcal{K}=\mathcal{K}(M)$. This is the canonical line bundle of the $\mathrm{CR}$ manifold $M$ with first Chern class $c_{1}(\mathcal{K}) \in H^{2}(M, \mathbb{Z})$. Its dual is the anticanonical bundle, denoted by $\mathcal{K}^{-1}$.

When dealing with a strictly pseudoconvex CR manifold, we will often assume the existence and choice of some $(m+2)$ nd root $\mathcal{E}(1)$ of the anticanonical bundle $\mathcal{K}^{-1}$, that is a complex line bundle over $M$ with

$$
\mathcal{E}(1)^{m+2}=\mathcal{K}^{-1} \text {. }
$$

The dual bundle of this root is denoted by $\mathcal{E}(-1)$. Then, for any integer $p \in \mathbb{Z}$, we have the $p$ th power $\mathcal{E}(p)$ of $\mathcal{E}(1)$. We call $p$ the weight of $\mathcal{E}(p)$. In particular, the canonical bundle $\mathcal{K}$ has weight $-(m+2)$, whereas the anticanonical bundle $\mathcal{K}^{-1}$ has weight $m+2$.

In general, the existence of an $(m+2)$ nd root $\mathcal{E}(1)$ is restrictive to the global nature of the underlying CR structure on $M$. For the application of tractor calculus in CR geometry this assumption is basic. In fact, the standard homogeneous model of CR geometry on the sphere allows a natural choice for $\mathcal{E}(1)$ (see [2]). For our treatment of spin ${ }^{\mathbb{C}}$ structures in $\mathrm{CR}$ geometry the choice of some $\mathcal{E}(1)$ is useful as well.

\section{Pseudo-Hermitian geometry}

Let $\left(M^{2 m+1}, H(M), J\right), m \geq 1$, be strictly pseudoconvex. Since $M$ is orientable, there exists some 1-form $\theta$ on $M$, whose kernel $\operatorname{Ker}(\theta)$ defines the contact distribution $H(M)$. The differential $\mathrm{d} \theta$ is a non-degenerate 2 -form on $H(M)$, and the conditions

$$
\iota_{T} \theta=\theta(T)=1 \quad \text { and } \quad \iota_{T} \mathrm{~d} \theta=0
$$

define a unique vector field $T=T_{\theta}$, which is the Reeb vector field of $\theta$. We use to call $T$ the characteristic vector. The tangent bundle $T(M)$ splits into the direct sum

$$
T(M)=H(M) \oplus \mathbb{R} T
$$


with corresponding projection $\pi_{\theta}: T(M) \rightarrow H(M)$. We use to say that vectors in $H(M)$ are transverse (to the characteristic direction of $T$ ). Note that $\mathfrak{L}_{T} \theta=\mathfrak{L}_{T} \mathrm{~d} \theta=0$ for the Lie derivatives with respect to $T_{\theta}$.

Furthermore,

$$
g_{\theta}(X, Y):=\frac{1}{2} \mathrm{~d} \theta(X, J Y), \quad X, Y \in H(M),
$$

defines a non-degenerate, symmetric bilinear form, i.e., a metric on $H(M)$, which is either positive or negative definite. In case $g_{\theta}$ is positive definite, we call $\theta \in \Omega^{1}(M)$ an adapted pseudoHermitian structure for $\left(M^{2 m+1}, H(M), J\right)$. Note that any two pseudo-Hermitian structures $\theta$ and $\tilde{\theta}$ differ only by some positive function or conformal scale, i.e., $\tilde{\theta}=\mathrm{e}^{2 f} \theta$ for some $f \in C^{\infty}(M)$.

Let us fix some adapted pseudo-Hermitian structure $\theta$ on $M$. To $\theta$ we have the WebsterTanaka connection $\nabla^{\mathrm{W}}$ on $T(M)$ (see $[21,22]$ ), for which by definition the characteristic vector $T$, the metric $g_{\theta}$ and the complex structure $J$ on $H(M)$ are parallel. Hence, the structure group of $\nabla^{\mathrm{W}}$ is the unitary group $\mathrm{U}(m)$. In characteristic direction, we have

$$
\nabla_{T}^{\mathrm{W}} X=\frac{1}{2}([T, X]-J[T, J X])
$$

for $X \in \Gamma(H(M))$.

The torsion is given by some obligatory part

$$
\nabla_{X}^{\mathrm{W}} Y-\nabla_{Y}^{\mathrm{W}} X-[X, Y]=\mathrm{d} \theta(X, Y) T
$$

with transverse $X, Y$ in $H(M)$ and, furthermore, by

$$
\operatorname{Tor}^{\mathrm{W}}(T, X)=-\frac{1}{2}([T, X]+J[T, J X]), \quad X \in H(M) .
$$

We call the latter part

$$
\tau(X):=\operatorname{Tor}^{\mathrm{W}}(T, X), \quad X \in H(M),
$$

Webster torsion tensor of $\theta$ on $(M, H(M), J)$. This is a symmetric and trace-free tensor. The composition $\tau \circ J=-J \circ \tau$ is symmetric and trace-free as well. We set $\tau(X, Y)=g_{\theta}(\tau X, Y)$, $X, Y \in H(M)$.

As usual the curvature operator $R^{\mathrm{W}}(X, Y)$ of $\nabla^{\mathrm{W}}$ is defined by

$$
R^{\mathrm{W}}(X, Y):=\nabla_{X}^{\mathrm{W}} \nabla_{Y}^{\mathrm{W}}-\nabla_{Y}^{\mathrm{W}} \nabla_{X}^{\mathrm{W}}-\nabla_{[X, Y]}^{\mathrm{W}}
$$

for any $X, Y \in T(M)$. Since $\nabla^{\mathrm{W}}$ is metric, $R^{\mathrm{W}}(X, Y)$ is skew-symmetric with respect to $g_{\theta}$ on $H(M)$. The first Bianchi identity for $X, Y, Z \in H(M)$ is given by the cyclic sum

$$
\sum_{X Y Z} R^{\mathrm{W}}(X, Y) Z=\sum_{X Y Z} \mathrm{~d} \theta(X, Y) \tau(Z) .
$$

For any $X \in T(M)$, the Webster-Ricci endomorphism $\mathrm{Ric}^{\mathrm{W}}(X)$ is the $g_{\theta}$-trace of $R(X, \cdot)(\cdot)$, and the Webster scalar curvature is the trace $\mathrm{scal}^{\mathrm{W}}=\operatorname{tr}_{\theta} \mathrm{Ric}^{\mathrm{W}}$ of the Webster-Ricci tensor on $H(M)$. On the other hand, the pseudo-Hermitian Ricci form is given by

$$
\rho_{\theta}(X, Y):=\frac{1}{2} \operatorname{tr}_{\theta}\left(g_{\theta}\left(R^{\mathrm{W}}(X, Y, J \cdot, \cdot)\right)\right)
$$

for any $X, Y \in H(M)$. Then we have

$$
\operatorname{Ric}^{\mathrm{W}}(X, Y)=\rho_{\theta}(X, J Y)+2(m-1) \tau(X, J Y)
$$


for any $X, Y \in H(M)$, where $\rho_{\theta}$ corresponds to the $J$-invariant and $\tau$ is the $J$-antiinvariant part of $\operatorname{Ric}^{\mathrm{W}}$ on $H(M)$.

If $m \geq 2$ and the pseudo-Hermitian Ricci form $\rho_{\theta}$ is a multiple of $\mathrm{d} \theta$ we call $\theta$ a pseudo-Einstein structure on the CR manifold $\left(M^{2 m+1}, H(M), J\right)$ (see [11]). For $m=1$ this condition is vacuous. However, for $m>1$ the pseudo-Einstein condition implies $\operatorname{Ric}^{\mathrm{W}}(T, J X)=\frac{1}{4 m} X\left(\mathrm{scal}^{\mathrm{W}}\right)$ for any $X \in H(M)$. This is a suitable replacement for the Einstein condition when $m=1$ (see [3]). In any case we have $\rho_{\theta}=\frac{\mathrm{scal}^{\mathrm{W}}}{4 m} \mathrm{~d} \theta$ and the Webster scalar curvature of some pseudo-Einstein structure $\theta$ need not be constant. In fact, it is constant if and only if

$$
\operatorname{Ric}^{\mathrm{W}}(T)=\operatorname{tr}_{\theta}\left(\nabla{ }^{\mathrm{W}} \tau\right)(\cdot)=0 .
$$

\section{Spin $^{\mathbb{C}}$ structures}

Recall that the group $\operatorname{Spin}^{\mathbb{C}}(2 m)$ is a central extension of $\mathrm{SO}(2 m)$ given by the exact sequence

$$
1 \rightarrow \mathbb{Z}_{2} \rightarrow \operatorname{Spin}^{\mathbb{C}}(2 m) \rightarrow \mathrm{SO}(2 m) \times \mathrm{U}(1) \rightarrow 1 .
$$

This gives a twisted product $\operatorname{Spin}^{\mathbb{C}}(2 m)=\operatorname{Spin}(2 m) \times_{\mathbb{Z}_{2}} \mathrm{U}(1)$ with the spin group. We have $\operatorname{Spin}^{\mathbb{C}}(2 m) / \mathrm{U}(1) \cong \mathrm{SO}(2 m)$, and a group homomorphism $\lambda: \operatorname{Spin}^{\mathbb{C}}(2 m) \rightarrow \mathrm{SO}(2 m)$ as well as $\operatorname{Spin}^{\mathbb{C}}(2 m) / \operatorname{Spin}(2 m) \cong \mathrm{U}(1)$. Note that there is also a canonical homomorphism

$$
j: \mathrm{U}(m) \rightarrow \operatorname{Spin}^{\mathbb{C}}(2 m),
$$

which is the lift of $\iota \times \operatorname{det}: \mathrm{U}(m) \rightarrow \mathrm{SO}(2 m) \times \mathrm{U}(1)$.

Now let $\theta$ be a pseudo-Hermitian form on the strictly pseudoconvex CR manifold $\left(M^{2 m+1}\right.$, $H(M), J), m \geq 1$. This gives rise to the metric $g_{\theta}$ on the Levi distribution $H(M)$. We denote by $\mathrm{SO}(H(M))$ the principal $\mathrm{SO}(2 m)$-bundle of orthonormal frames in $H(M)$. A spin ${ }^{\mathbb{C}}$ structure to $\theta$ on $M$ is a reduction $(P, \Lambda)$ of the frame bundle $\mathrm{SO}(H(M))$. This means here, $P \rightarrow M$ is some principal $\operatorname{Spin}^{\mathbb{C}}(2 m)$-bundle with fiber bundle map $\Lambda: P \rightarrow \mathrm{SO}(H(M))$ such that $\Lambda(p \cdot s)=$ $\Lambda(p) \cdot \lambda(s)$ for all $p \in P$ and $s \in \operatorname{Spin}^{\mathbb{C}}(2 m)$.

Let $(P, \Lambda)$ be some fixed $\operatorname{spin}^{\mathbb{C}}$ structure for $(M, \theta)$. Then $P_{1}:=P / \operatorname{Spin}(2 m) \rightarrow M$ is a principal $\mathrm{U}(1)$-bundle, and we denote the associated complex line bundle by $L \rightarrow M$. This is the determinant bundle of the $\operatorname{spin}^{\mathbb{C}}$ structure. The corresponding fiber bundle map $\Lambda_{1}: P \rightarrow$ $\mathrm{SO}(H(M)) \times P_{1}$ over $M$ is a twofold covering. On the other hand, let $L(\beta) \rightarrow M$ be a complex line bundle determined by some integral class $\beta \in H^{2}(M, \mathbb{Z})$. Then, if

$$
\beta \equiv-c_{1}(\mathcal{K}) \bmod 2,
$$

there exists a $\operatorname{spin}^{\mathbb{C}}$ structure $(P, \Lambda)$ to $\theta$ on $M$ with determinant bundle $L(\beta)$.

There exists always the canonical spin $^{\mathbb{C}}$ structure to $\theta$ on $M$, which stems from the lift $j: \mathrm{U}(m) \rightarrow \operatorname{Spin}^{\mathbb{C}}(2 m)$. The corresponding determinant bundle is $\mathcal{K}^{-1}$. All other spin ${ }^{\mathbb{C}}$ structures differ from the canonical one by multiplication with a principal $\mathrm{U}(1)$-bundle, related to some line bundle $E(\alpha), \alpha \in H^{2}(M, \mathbb{Z})$. The corresponding determinant bundle $L(\beta)$ satisfies $E(\alpha)^{2}=\mathcal{K} \otimes L(\beta)$. Spin ${ }^{\mathbb{C}}$ structures with the same determinant bundle $L(\beta)$ are parametrized by the elements in $H^{1}\left(M, \mathbb{Z}_{2}\right)$ (see $\left.[10,18]\right)$.

In particular, if $c_{1}(\mathcal{K}) \equiv 0 \bmod 2$, then $\theta$ on $M$ admits some $\operatorname{spin}^{\mathbb{C}}$ structure with trivial determinant bundle. This represents an ordinary spin structure for the Levi distribution $H(M)$ with metric $g_{\theta}$ (cf. [12]). More generally, let us consider the powers $\mathcal{E}(p), p \in \mathbb{Z}$, of an $(m+2)$ nd root $\mathcal{E}(1)$ of $\mathcal{K}^{-1}$. Then $-c_{1}(\mathcal{K})=(m+2) c_{1}(\mathcal{E}(1))$, and a $\operatorname{spin}^{\mathbb{C}}$ structure for $(M, \theta)$ with determinant bundle $L=\mathcal{E}(p)$ exists when

$$
(m+2-p) c_{1}(\mathcal{E}(1)) \equiv 0 \quad \bmod 2 .
$$


Lemma 4.1. Let $\mathcal{E}(1)$ be an $(m+2) n d$ root of $\mathcal{K}^{-1} \rightarrow M^{2 m+1}$. Then $\theta$ on $M$ admits a $\operatorname{spin}^{\mathbb{C}}$ structure with determinant bundle $L=\mathcal{E}(p), p \in \mathbb{Z}$, if

(i) $\mathcal{E}(1)$ itself admits some square root, or

(ii) $m$ and $p \in \mathbb{Z}$ are odd, or

(iii) $m$ and $p$ are even.

We say that a $\operatorname{spin}^{\mathbb{C}}$ structure with determinant bundle $L=\mathcal{E}(p)$ has weight $p$. In the following we assume that $\operatorname{spin}^{\mathbb{C}}$ structures to $\theta$ on $M$ exist for all necessary weights $p \in \mathbb{Z}$.

\section{$5 \quad$ Spinors and connections}

Let $\left(M^{2 m+1}, H(M), J\right)$ be a strictly pseudoconvex CR manifold of hypersurface type and CR dimension $m \geq 1$, and let $(P, \Lambda)$ be a spin ${ }^{\mathbb{C}}$ structure of weight $\ell \in \mathbb{Z}$ for some given pseudoHermitian form $\theta$ on $M$. The choice of $(P, \Lambda)$ gives rise to an associated spinor bundle

$$
\Sigma(H(M)):=P \times_{\rho_{2 m}} \Sigma
$$

over $M$, where $\rho_{2 m}$ denotes the representation of $\operatorname{Spin}^{\mathbb{C}}(2 m)$ on the complex spinor module $\Sigma$. Note that the center $\mathrm{U}(1)$ acts by complex scalar multiplication on $\Sigma$. The spinor bundle has $\operatorname{rk}_{\mathbb{C}}\left(\Sigma(H(M))=2^{m}\right.$.

The spinor bundle $\Sigma(H(M))$ is equipped with a Hermitian inner product $\langle\cdot, \cdot\rangle$, and we have a Clifford multiplication

$$
\begin{aligned}
c: H(M) \otimes \Sigma(H(M)) & \rightarrow \Sigma(H(M)), \\
(X, \phi) & \mapsto X \cdot \phi,
\end{aligned}
$$

which satisfies

$$
\langle X \cdot \psi, \phi\rangle=-\langle\psi, X \cdot \phi\rangle
$$

for any transverse $X \in H(M)$ and $\phi \in \Sigma(H(M))$, given at some point of $M$. The multiplication $c$ extends to the complex Clifford bundle $\mathbb{C l}(H(M))$ of the Levi distribution.

The Webster-Tanaka connection $\nabla^{\mathrm{W}}$ to $\theta$ stems from a principal fiber bundle connection on the unitary frame bundle, contained in $\mathrm{SO}(H(M))$. This gives rise to a covariant derivative $\nabla^{\mathrm{W}}$ on any root of $\mathcal{K}^{-1}$ and its powers, in particular, for $\mathcal{E}(1)$ and the determinant bundle $L=\mathcal{E}(\ell)$.

Recall that $(P, \Lambda)$ induces a twofold covering map $P \rightarrow \mathrm{SO}(H(M)) \times P_{1}$. Then the WebsterTanaka connection lifts to $P$, which in turn gives rise to some covariant derivative on spinor fields:

$$
\begin{aligned}
\nabla^{\Sigma}: \Gamma(T(M)) \otimes \Gamma(\Sigma(H(M)) & \rightarrow \Gamma(\Sigma(H(M)), \\
(X, \phi) & \mapsto \nabla_{X}^{\Sigma} \phi .
\end{aligned}
$$

Note that this construction does not need an auxiliary connection on the determinant bundle $L$. We only use the Webster-Tanaka connection on $L$ and call $\nabla^{\Sigma}$ the Webster-Tanaka spinor derivative to the given $\operatorname{spin}^{\mathbb{C}}$ structure of weight $\ell$.

The spinor derivative satisfies the rules

$$
\nabla_{Y}^{\Sigma}(X \cdot \phi)=\left(\nabla_{Y}^{\mathrm{W}} X\right) \cdot \phi+X \cdot \nabla_{Y}^{\Sigma} \phi \quad \text { and } \quad Y\langle\phi, \psi\rangle=\left\langle\nabla_{Y}^{\Sigma} \phi, \psi\right\rangle+\left\langle\phi, \nabla_{Y}^{\Sigma} \psi\right\rangle
$$


for any $X \in \Gamma(H(M)), Y \in \Gamma(T(M))$ and $\phi, \psi \in \Gamma(\Sigma(H(M)))$. Locally, with respect to some orthonormal frame $s=\left(s_{1}, \ldots, s_{2 m}\right)$, the spinor derivative is given by the formula

$$
\nabla^{\Sigma} \phi=\mathrm{d} \phi+\frac{1}{2} \sum_{j<k}^{2 m} g_{\theta}\left(\nabla^{\mathrm{W}} s_{j}, s_{k}\right) s_{j} s_{k} \cdot \phi+\frac{1}{2} A^{\mathrm{W}, s} \phi,
$$

where $A^{\mathrm{W}, s}$ denotes the local Webster-Tanaka connection form on $P_{1}$ with values in $\mathrm{i} \mathbb{R}$. The curvature $R^{\Sigma}$ of the spinor derivative $\nabla^{\Sigma}$ is then given by

$$
\begin{aligned}
R^{\Sigma}(X, Y) \phi & =\nabla_{X}^{\Sigma} \nabla_{Y}^{\Sigma} \phi-\nabla_{Y}^{\Sigma} \nabla_{X}^{\Sigma} \phi-\nabla_{[X, Y]}^{\Sigma} \phi \\
& =\frac{1}{4} \sum_{j, k=1}^{2 m} g_{\theta}\left(R^{\mathrm{W}}(X, Y) s_{j}, s_{k}\right) s_{j} s_{k} \cdot \phi+\frac{1}{2} \mathrm{~d} A^{\mathrm{W}}(X, Y) \phi
\end{aligned}
$$

for any $X, Y \in T M$ and spinor $\phi \in \Gamma(\Sigma(H(M)))$. Note that

$$
\mathrm{d} A^{\mathrm{W}}=\frac{-\mathrm{i} \ell}{m+2} \rho_{\theta}
$$

is a multiple of the pseudo-Hermitian Ricci form $\rho_{\theta}$.

The underlying pseudo-Hermitian form $\theta$ gives rise to further structure on the spinor bundle $\Sigma(H(M))$. In fact, recall that $\mathrm{d} \theta$ is $\nabla^{\mathrm{W}}$-parallel and basic, i.e., $\iota_{T} \mathrm{~d} \theta=0$. We set $\Theta:=\frac{\mathrm{i} \theta}{2} \in$ $\mathbb{C l}(H(M))$ in the complex Clifford bundle. Then $\Theta$ acts by real eigenvalues $\mu_{q}=m-2 q$, $q \in\{0, \ldots, m\}$ on $\Sigma(H(M))$. We obtain the decomposition

$$
\Sigma(H(M))=\bigoplus_{q=0}^{m} \Sigma^{\mu_{q}}(H(M))
$$

into $\Theta$-eigenspaces $\Sigma^{\mu_{q}}(H(M))$ of rank $\left(\begin{array}{c}m \\ q\end{array}\right)$ to the eigenvalue $\mu_{q}$ (see [18]). We call the bundles to the $\Theta$-eigenvalues $\mu_{q}= \pm m$ extremal. (We also define $\Sigma^{a}=\{0\}$ to be trivial for any $a>m$ and $a<-m$.) Accordingly, we can decompose any spinor $\phi$ on $M$ into

$$
\phi=\sum_{q=0}^{m} \phi_{\mu_{q}}
$$

where $\Theta \phi_{\mu_{q}}=(m-2 q) \phi_{\mu_{q}}$. This decomposition is compatible with the spinor derivative $\nabla^{\Sigma}$.

\section{Kohn-Dirac and twistor operators}

Let $\left(M^{2 m+1}, H(M), J\right), m \geq 1$, be strictly pseudoconvex. We have $H(M) \otimes \mathbb{C}=T_{10} \oplus T_{01}$ and any real transverse vector $X \in H(M)$ can be written as $X=X_{10}+X_{01}$ with

$$
X_{10}=\frac{X-\mathrm{i} J X}{2} \in T_{10} \quad \text { and } \quad X_{01}=\frac{X+\mathrm{i} J X}{2} \in T_{01} .
$$

If $e=\left(e_{1}, \ldots, e_{m}\right)$ denotes a complex orthonormal basis of $\left(H, J, g_{\theta}\right)$, i.e., $s=\left(e_{1}, J e_{1}, \ldots, e_{m}\right.$, $\left.J e_{m}\right)$ is a real orthonormal basis of $\left(H, g_{\theta}\right)$, we set

$$
E_{\alpha}:=\left(e_{\alpha}\right)_{10}=\frac{e_{\alpha}-\mathrm{i} J e_{\alpha}}{2}, \quad \alpha=1, \ldots, m .
$$


The vectors $\left(E_{1}, \ldots, E_{m}\right)$ form an orthogonal basis with respect to the Levi form on $T_{10}$. As elements in the complexified Clifford algebra $\mathbb{C l}(H(M))$ we have $E_{\alpha} E_{\alpha}=0$ and $E_{\alpha} \overline{E_{\beta}}+\overline{E_{\beta}} E_{\alpha}=$ $-\delta_{\alpha \beta}$ for any $\alpha, \beta=1, \ldots, m$. Moreover,

$$
\sum_{\alpha=1}^{m} \overline{E_{\alpha}} E_{\alpha}=-\frac{1}{2}(m+\Theta), \quad \sum_{\alpha=1}^{m} E_{\alpha} \overline{E_{\alpha}}=-\frac{1}{2}(m-\Theta) .
$$

Now let $\theta$ be a pseudo-Hermitian form on $M$ with $\operatorname{spin}^{\mathbb{C}}$ structure of weight $\ell \in \mathbb{Z}$. The spinorial derivative $\nabla^{\Sigma}$ on $\Sigma(H(M))$ is induced by the Webster-Tanaka connection. In the following, we allow covariant derivatives with respect to $Z \in H(M) \otimes \mathbb{C}$. This is defined by $\mathbb{C}$-linear extension and denoted by $\nabla_{Z}^{\text {tr }}$. This derivative in transverse direction decomposes into

$$
\nabla^{\operatorname{tr}}=\nabla_{10} \oplus \nabla_{01}
$$

i.e., for any spinor $\phi \in \Gamma(\Sigma(H(M)))$, we have locally

$$
\nabla_{10} \phi=\sum_{\alpha=1}^{m} E_{\alpha}^{*} \otimes \nabla_{E_{\alpha}}^{\operatorname{tr}} \phi \quad \text { and } \quad \nabla_{01} \phi=\sum_{\alpha=1}^{m}{\overline{E_{\alpha}}}^{*} \otimes \nabla_{E_{\alpha}}^{\operatorname{tr}} \phi
$$

with respect to some frame $\left(E_{1}, \ldots, E_{m}\right)$ of $T_{10}$.

Recall that Clifford multiplication is denoted by $c$. Then we can define the first order differential operators

$$
D_{-} \phi=c\left(\nabla_{10} \phi\right) \quad \text { and } \quad D_{+} \phi=c\left(\nabla_{01} \phi\right)
$$

for spinors $\phi \in \Gamma(\Sigma(H(M)))$. Locally, the two operators are given by

$$
D_{-} \phi=2 \sum_{\alpha=1}^{m} \overline{E_{\alpha}} \cdot \nabla_{E_{\alpha}}^{\operatorname{tr}} \phi \quad \text { and } \quad D_{+} \phi=2 \sum_{\alpha=1}^{m} E_{\alpha} \cdot \nabla_{E_{\alpha}}^{\operatorname{tr}} \phi .
$$

Note that $\Theta \cdot X_{10}-X_{10} \cdot \Theta=-2 X_{10}$ for $X_{10} \in T_{10}$. This shows

$$
T_{10} \cdot \Sigma^{\mu_{q}} \subseteq \Sigma^{\mu_{q+1}} \quad \text { and } \quad T_{01} \cdot \Sigma^{\mu_{q}} \subseteq \Sigma^{\mu_{q-1}}
$$

for any $q \in\{0, \ldots, m\}$. Hence, the operator $D_{+}$maps spinors from $\Gamma\left(\Sigma^{\mu_{q}}\right)$ to $\Gamma\left(\Sigma^{\mu_{q+1}}\right)$. Similarly, $D_{-}: \Gamma\left(\Sigma^{\mu_{q}}\right) \rightarrow \Gamma\left(\Sigma^{\mu_{q-1}}\right)$. In fact, we have $\left[\Theta, D_{+}\right]=-2 D_{+}$and $\left[\Theta, D_{-}\right]=2 D_{-}$.

We compute the square of $D_{+}$. Locally, around any $p \in M$, we can choose a synchronized frame of the form $\left(e_{1}, \ldots, e_{m}\right)$ with

$$
\nabla_{e_{\alpha}}^{\mathrm{W}} e_{\beta}(p)=0, \quad \alpha, \beta \in\{1, \ldots, m\} .
$$

Then

$$
\begin{aligned}
\left(D_{+}\right)^{2} \phi & =4 \sum_{\alpha, \beta=1}^{m} E_{\alpha} E_{\beta} \nabla_{\overline{E_{\alpha}}}^{\operatorname{tr}} \nabla_{\overline{E_{\beta}}}^{\operatorname{tr}} \phi=2 \sum_{\alpha, \beta} E_{\alpha} E_{\beta} \cdot R^{\Sigma}\left(\overline{E_{\alpha}}, \overline{E_{\beta}}\right) \phi \\
& =-2\left(\sum_{\alpha, \beta} E_{\alpha} \tau\left(\overline{E_{\alpha}}\right) E_{\beta} \overline{E_{\beta}}+E_{\alpha} \overline{E_{\alpha}} E_{\beta} \tau\left(\overline{E_{\beta}}\right)\right) \phi=0,
\end{aligned}
$$

where we use (3.1), (5.1) and the fact that $\tau, \tau \circ J$ are trace-free. Similarly, we obtain $D_{-}^{2}=0$. Thus, we have constructed two chain complexes

$$
0 \rightarrow \Gamma\left(\Sigma^{\mu_{0}}\right) \stackrel{D_{+}}{\longrightarrow} \Gamma\left(\Sigma^{\mu_{1}}\right) \stackrel{D_{+}}{\longrightarrow} \cdots \stackrel{D_{+}}{\longrightarrow} \Gamma\left(\Sigma^{\mu_{m-1}}\right) \stackrel{D_{+}}{\longrightarrow} \Gamma\left(\Sigma^{\mu_{m}}\right) \rightarrow 0
$$


and

$$
0 \rightarrow \Gamma\left(\Sigma^{\mu_{m}}\right) \stackrel{D_{-}}{\longrightarrow} \Gamma\left(\Sigma^{\mu_{m-1}}\right) \stackrel{D_{-}}{\longrightarrow} \cdots \stackrel{D_{-}}{\longrightarrow} \Gamma\left(\Sigma^{\mu_{1}}\right) \stackrel{D_{-}}{\longrightarrow} \Gamma\left(\Sigma^{\mu_{0}}\right) \rightarrow 0 .
$$

From the discussions in Section 10 it will become clear that these complexes produce finite dimensional cohomology groups. This compares to the construction of spinorial cohomology on Kähler manifolds as described in [15].

Next we define

$$
D_{\theta} \phi=c\left(\nabla^{\operatorname{tr}} \phi\right)=\left(D_{+}+D_{-}\right) \phi .
$$

This is a first order, subelliptic differential operator acting on spinor fields $\phi \in \Gamma(\Sigma(H(M))$. We call $D_{\theta}$ the Kohn-Dirac operator to $\theta$ with spin $^{\mathbb{C}}$ structure of weight $\ell$ on $M$ (see [18]; cf. [12, 20]). Locally, with respect to an orthonormal frame $\left(s_{1}, \ldots, s_{m}\right)$, the Kohn-Dirac operator is given by

$$
D_{\theta} \phi=\sum_{i=1}^{2 m} s_{i} \cdot \nabla_{s_{i}}^{\mathrm{tr}} \phi .
$$

Obviously, $D_{\theta}$ does not preserve the decomposition of spinors with respect to $\Theta$-eigenvalues. However, we have the identity

$$
D_{\theta}^{2}=D_{+} D_{-}+D_{-} D_{+},
$$

which shows that the square of the Kohn-Dirac operator maps sections of $\Sigma^{\mu_{q}}(H(M))$ to sections of $\Sigma^{\mu_{q}}(H(M))$ again, i.e.,

$$
D_{\theta}^{2}: \Gamma\left(\Sigma^{\mu_{q}}\right) \rightarrow \Gamma\left(\Sigma^{\mu_{q}}\right), \quad q=0, \ldots, m .
$$

On the spinor bundle, we have the $L_{2}$-inner product defined by

$$
(\phi, \psi):=\int_{M}\langle\phi, \psi\rangle \operatorname{vol}_{\theta}
$$

for compactly supported spinors $\phi, \psi \in \Gamma_{c}(\Sigma)$, where

$$
\operatorname{vol}_{\theta}:=\theta \wedge(\mathrm{d} \theta)^{m}
$$

denotes the induced volume form of the pseudo-Hermitian structure $\theta$ on $M$. The Kohn-Dirac operator $D_{\theta}$ is formally self-adjoint with respect to this $L_{2}$-inner product $(\cdot, \cdot)$ on $\Gamma_{c}(\Sigma)$ (see [12]).

Complementary to the Kohn-Dirac operator $D_{\theta}$, we have twistor operators $P^{\left(\mu_{q}\right)}$ acting on $\Gamma\left(\Sigma^{\mu_{q}}(H(M))\right)$ for $q=0, \ldots, m$. In fact, there are orthogonal decompositions

$$
T_{10}^{*} \otimes \Sigma^{\mu_{q}} \cong \operatorname{Ker}(c) \oplus \Sigma^{\mu_{q-1}} \quad \text { and } \quad T_{01}^{*} \otimes \Sigma^{\mu_{q}} \cong \operatorname{Ker}(c) \oplus \Sigma^{\mu_{q+1}},
$$

where $\operatorname{Ker}(c)$ denote the corresponding kernels of the Clifford multiplication. Then with

$$
a_{q}:=\frac{1}{2(q+1)} \quad \text { and } \quad b_{q}:=\frac{1}{2(m-q+1)}
$$

we have for the derivatives $\nabla_{10} \phi_{\mu_{q}}$ and $\nabla_{01} \phi_{\mu_{q}}$ of a spinor $\phi_{\mu_{q}} \in \Gamma\left(\Sigma^{\mu_{q}}\right)$ the decompositions

$$
\begin{aligned}
& \nabla_{10} \phi_{\mu_{q}}=P_{10} \phi_{\mu_{q}}-b_{q} \sum_{\alpha=1}^{m} E_{\alpha}^{*} \otimes E_{\alpha} \cdot D_{-} \phi_{\mu_{q}}, \\
& \nabla_{01} \phi_{\mu_{q}}=P_{01} \phi_{\mu_{q}}-a_{q} \sum_{\alpha=1}^{m}{\overline{E_{\alpha}}}^{*} \otimes \overline{E_{\alpha}} \cdot D_{+} \phi_{\mu_{q}},
\end{aligned}
$$


where the twistor operators map to $\operatorname{Ker}(c)$ by

$$
\begin{aligned}
& P_{10}\left(\phi_{\mu_{q}}\right)=\sum_{\alpha=1}^{m} E_{\alpha}^{*} \otimes\left(\nabla_{E_{\alpha}} \phi_{\mu_{q}}+b_{q} E_{\alpha} \cdot D_{-} \phi_{\mu_{q}}\right), \\
& P_{01}\left(\phi_{\mu_{q}}\right)=\sum_{\alpha=1}^{m}{\overline{E_{\alpha}}}^{*} \otimes\left(\nabla_{\overline{E_{\alpha}}} \phi_{\mu_{q}}+a_{q} \overline{E_{\alpha}} \cdot D_{+} \phi_{\mu_{q}}\right),
\end{aligned}
$$

respectively. The sum $P^{\left(\mu_{q}\right)}=P_{10}+P_{01}$ is given with respect to a local orthonormal frame $s$ by

$$
P^{\left(\mu_{q}\right)} \phi_{\mu_{q}}=\sum_{i=1}^{2 m} s_{i}^{*} \otimes\left(\nabla_{s_{i}}^{\operatorname{tr}} \phi_{\mu_{q}}+a_{q} \frac{s_{i}+\mathrm{i} J s_{i}}{2} D_{+} \phi_{\mu_{q}}+b_{q} \frac{s_{i}-\mathrm{i} J s_{i}}{2} D_{-} \phi_{\mu_{q}}\right) .
$$

This is the projection of $\nabla^{\operatorname{tr}} \phi_{\mu_{q}}$ to the kernel $\operatorname{Ker}(c)$.

\section{Covariant components and spinorial CR invariants}

In the previous section we have introduced Kohn-Dirac operators $D_{\theta}$ and twistor operators $P^{\left(\mu_{q}\right)}$ for $\operatorname{spin}^{\mathbb{C}}$ structures of weight $\ell \in \mathbb{Z}$. We have only used the Webster-Tanaka connection for their construction. Now we compute the transformation law for $D_{\theta}$ and $P^{\left(\mu_{q}\right)}$ under conformal change of the pseudo-Hermitian structure. It turns out that certain components of $D_{\theta}$ and $P^{\left(\mu_{q}\right)}$ are CR invariants.

Let $\theta$ and $\tilde{\theta}=\mathrm{e}^{2 f} \theta$ be two adapted pseudo-Hermitian structures on $\left(M^{2 m+1}, H(M), J\right)$, $m \geq 1$. We denote by $\nabla^{\mathrm{W}}$ and $\nabla^{\Sigma}$ derivatives with respect to $\theta$. The derivatives with respect to $\tilde{\tilde{\theta}}$ are simply denote by $\widetilde{\nabla}$. Note that the structure group of the Webster-Tanaka connection is $\mathrm{U}(m)$ for any pseudo-Hermitian form. We have the transformation rule

$$
\begin{aligned}
& \widetilde{\nabla}_{X_{10}} Y=\nabla_{X_{10}}^{\mathrm{W}} Y+2 X_{10}(f) Y_{10}+2 Y_{10}(f) X_{10}-2 g_{\theta}\left(X_{10}, Y_{01}\right) \operatorname{grad}_{01}(f), \\
& \widetilde{\nabla}_{X_{01}} Y=\nabla_{X_{01}}^{\mathrm{W}} Y+2 X_{01}(f) Y_{01}+2 Y_{01}(f) X_{01}-2 g_{\theta}\left(X_{01}, Y_{10}\right) \operatorname{grad}_{10}(f),
\end{aligned}
$$

where $X=X_{10}+X_{01}$ and $Y=Y_{10}+Y_{01}$ are transverse vectors (see, e.g., [11]). The gradient $\operatorname{grad}_{\theta}(f) \in \Gamma(H(M))$ with complex components $\operatorname{grad}_{10}(f) \in \Gamma\left(T_{10}\right)$ and $\operatorname{grad}_{01}(f) \in \Gamma\left(T_{01}\right)$ is dual via $g_{\theta}$ to the restriction of the differential $\mathrm{d} f$ to $H(M)$.

Now let $(P, \Lambda)$ be some $\operatorname{spin}^{\mathbb{C}}$ structure of weight $\ell \in \mathbb{Z}$ to $\theta$ on $\left(M^{2 m+1}, H(M), J\right)$. The canonical bundle $\mathcal{K}$ and all line bundles $\mathcal{E}(p), p \in \mathbb{Z}$, are natural for the underlying CR structure. In particular, the determinant bundle $L \rightarrow M$ of weight $\ell$ is natural, and the corresponding principal $\mathrm{U}(1)$-bundles $P_{1}$ and $\tilde{P}_{1}$ of frames in $L$ with respect to $\theta$ and $\tilde{\theta}$, respectively, are naturally identified. The same is true for the orthonormal frames in $H(M)$ to $\theta$ and $\tilde{\theta}$. Thus, there exists a unique $\operatorname{spin}^{\mathbb{C}}$ structure $(\tilde{P}, \tilde{\Lambda})$ with respect to $\tilde{\theta}$ on $M$, whose spinor frames are naturally identified with those of $(P, \Lambda)$. Of course, the determinant bundle to $(\tilde{P}, \tilde{\Lambda})$ has weight $\ell$ again, and there exists an unitary isomorphism

$$
\begin{aligned}
\Sigma(H(M)) & \cong \widetilde{\Sigma}(H(M)), \\
\phi & \mapsto \tilde{\phi},
\end{aligned}
$$

between the two kinds of spinor bundles such that $X \cdot \phi$ is sent to $\mathrm{e}^{-f} X^{\sim} \tilde{\phi}$ for any transverse vector $X \in H(M)$ and spinor $\phi \in \Sigma(H(M))$. Also note that $\widetilde{\Theta} \tilde{\phi}=(\Theta \phi) \tilde{)^{2}}$, i.e., the decomposition $\Sigma(H(M))=\oplus_{q=0}^{m} \Sigma^{\mu_{q}}(H(M))$ into $\Theta$-eigenspaces is CR-invariant.

We compare now the spinor derivatives with respect to $\theta$ and $\tilde{\theta}$, respectively. First, let $\sigma=E_{1} \wedge \cdots \wedge E_{m}$ be a local section in $\mathcal{K}^{-1} \rightarrow M$ and $\tilde{\sigma}=\widetilde{E_{1}} \wedge \cdots \wedge \widehat{E_{m}}$ the corresponding 
section with respect to $\tilde{\theta}$. Then $\tilde{\sigma}=\mathrm{e}^{-m f} \sigma$ and with (7.1) we obtain the transformation rule

$$
\begin{aligned}
& \widetilde{\nabla}_{X_{10}} \tilde{\sigma}=\left(A^{\sigma}\left(X_{10}\right)+(m+2) X_{10}(f)\right) \tilde{\sigma}=A^{\tilde{\sigma}}\left(X_{10}\right) \tilde{\sigma}, \\
& \widetilde{\nabla}_{X_{01}} \tilde{\sigma}=\left(A^{\sigma}\left(X_{01}\right)-(m+2) X_{01}(f)\right) \tilde{\sigma}=A^{\tilde{\sigma}}\left(X_{01}\right) \tilde{\sigma},
\end{aligned}
$$

$X=X_{10}+X_{01}$, for the local connections forms of $\mathcal{K}^{-1}$. This gives

$$
A^{\tilde{\sigma}}(X)-A^{\sigma}(X)=-\mathrm{i}(m+2)(J X)(f), \quad X \in H(M) .
$$

Accordingly,

$$
A^{\tilde{\sigma}}(X)-A^{\sigma}(X)=-\mathrm{i} \ell(J X)(f), \quad X \in H(M),
$$

for the local connection forms on $L=\mathcal{E}(\ell)$. With formulas in [12] we obtain for spinors $\phi$ the transformation rule

$$
\begin{aligned}
& \widetilde{\nabla}_{X_{10}} \tilde{\phi}=\widetilde{\nabla_{X_{10}}^{\Sigma} \phi}-\left(X_{10} \cdot \operatorname{grad}_{01}(f) \cdot \phi \tilde{)}+\frac{\ell-2}{2} X_{10}(f) \tilde{\phi}-\frac{1}{2} X_{10}(f)(\Theta \phi),\right. \\
& \widetilde{\nabla}_{X_{01}} \tilde{\phi}=\widetilde{\nabla_{X_{01}}^{\Sigma} \phi}-\left(X_{01} \cdot \operatorname{grad}_{10}(f) \cdot \phi \tilde{)}-\frac{\ell+2}{2} X_{01}(f) \tilde{\phi}+\frac{1}{2} X_{01}(f)(\Theta \phi) \tilde{r} .\right.
\end{aligned}
$$

This gives for $\phi_{\mu_{q}} \in \Gamma\left(\Sigma^{\mu_{q}}\right), q \in\{0, \ldots, m\}$,

$$
\begin{aligned}
& \tilde{D}_{-} \tilde{\phi}_{\mu_{q}}=\mathrm{e}^{-f}\left(D_{-} \phi_{\mu_{q}}+\left(m+1+\frac{\mu_{q}+\ell}{2}\right) \operatorname{grad}_{01}(f) \phi_{\mu_{q}}\right)^{\sim}, \\
& \tilde{D}_{+} \tilde{\phi}_{\mu_{q}}=\mathrm{e}^{-f}\left(D_{+} \phi_{\mu_{q}}+\left(m+1-\frac{\mu_{q}+\ell}{2}\right) \operatorname{grad}_{10}(f) \phi_{\mu_{q}}\right)^{\sim},
\end{aligned}
$$

and we obtain

$$
\begin{aligned}
& \tilde{D}_{-}\left(\mathrm{e}^{-v_{-} f} \tilde{\phi}_{\mu_{q}}\right)=\mathrm{e}^{-\left(v_{-}+1\right) f} \widetilde{D_{-} \phi_{\mu_{q}}} \quad \text { for }
\end{aligned}
$$

Hence, for the $\Theta$-eigenvalue $\mu_{q}=-\ell$, we have

$$
D_{\tilde{\theta}}\left(\mathrm{e}^{-(m+1) f} \tilde{\phi}_{-\ell}\right)=\mathrm{e}^{-(m+2) f} \widetilde{D_{\theta} \phi_{-\ell}},
$$

i.e., the restriction of the Kohn-Dirac operator $D_{\theta}$ of weight $\ell$ to $\Gamma\left(\Sigma^{-\ell}\right)$ acts CR-covariantly.

Recall that the given $\operatorname{spin}^{\mathbb{C}}$ structure of weight $\ell \in \mathbb{Z}$ on $M$ is determined by some complex line bundle $E(\alpha), \alpha \in H^{2}(M, \mathbb{Z})$.

Definition 7.1. Let $\left(M^{2 m+1}, H(M), J\right), m \geq 1$, be strictly pseudoconvex with pseudo-Hermitian form $\theta$ and $\operatorname{spin}^{\mathbb{C}}$ structure of weight $\ell \in \mathbb{Z}$.

(a) A spinor $\phi \in \Gamma\left(\Sigma(H(M))\right.$ in the kernel of the Kohn-Dirac operator, i.e., $D_{\theta} \phi=0$, is called harmonic. We denote by $\mathcal{H}^{q}(\alpha)$ the space of harmonic spinors with $\Theta$-eigenvalue $\mu_{q}$, $q \in\{0, \ldots, m\}$. Its dimension is denoted $h_{q}(\alpha)$.

(b) For weight $\ell \in\{-m,-m+2, \ldots, m-2, m\}$ the differential operator

$$
\mathcal{D}_{\ell}: \Gamma\left(\Sigma^{-\ell}\right) \rightarrow \Gamma\left(\Sigma^{-\ell+2}\right) \oplus \Gamma\left(\Sigma^{-\ell-2}\right)
$$

denotes the restriction of $D_{\theta}$ (of weight $\ell$ ) to spinors of $\Theta$-eigenvalue $-\ell$. We call $\mathcal{D}_{\ell}$ the eth (CR-covariant) component of the Kohn-Dirac operator.

(c) A spinor $\phi$ in the kernel of $\mathcal{D}_{\ell}$ is called harmonic of weight $\ell$.

Since $\mathcal{D}_{\ell}$ acts by (7.2) CR-covariantly, harmonic spinors of weight $\ell$ are CR invariants of $(H(M), J)$ on $M$. The dimension $h_{\frac{m+\ell}{2}}(\alpha)$ is a CR invariant as well. In Section 10 we will see that in fact all dimensions $h_{q}(\alpha), 0<q<m$, are CR-invariant numbers. 
Let us consider the twistor operators $P_{10}$ and $P_{01}$. Calculating as above we find

$$
\widetilde{\nabla}_{X_{10}}\left(\mathrm{e}^{-w_{-} f} \tilde{\phi}_{\mu_{q}}\right)+b_{q} X_{10} \tilde{D}_{-}\left(\mathrm{e}^{-w_{-} f} \tilde{\phi}_{\mu_{q}}\right)=\mathrm{e}^{-w_{-} f} \cdot\left(\nabla_{X_{10}} \phi_{\mu_{q}}+b_{q} X_{10} D_{-} \phi_{\mu_{q}}\right) \tilde{}
$$

exactly for $w_{-}=\frac{\ell-\mu_{q}}{2}-1$, and

$$
\widetilde{\nabla}_{X_{01}}\left(\mathrm{e}^{-w_{+} f} \tilde{\phi}_{\mu_{q}}\right)+a_{q} X_{01} \tilde{D}_{+}\left(\mathrm{e}^{-w_{+} f} \tilde{\phi}_{\mu_{q}}\right)=\mathrm{e}^{-w_{+} f} \cdot\left(\nabla_{X_{01}} \phi_{\mu_{q}}+a_{q} X_{01} D_{-} \phi_{\mu_{q}}\right) \tilde{T}
$$

exactly for $w_{+}=\frac{\mu_{q}-\ell}{2}-1$. Hence, the twistor operator $P^{\left(\mu_{q}\right)}=P_{10}+P_{01}$ is CR-covariant for the $\Theta$-eigenvalue $\mu_{q}=\ell$.

Definition 7.2. Let $\left(M^{2 m+1}, H(M), J\right), m \geq 1$, be strictly pseudoconvex with pseudo-Hermitian form $\theta$ and $\operatorname{spin}^{\mathbb{C}}$ structure of weight $\ell \in \mathbb{Z}$.

(a) For weight $\ell \in\{-m,-m+2, \ldots, m-2, m\}$ the differential operator

$$
\mathcal{P}_{\ell}: \Gamma\left(\Sigma^{\ell}\right) \rightarrow \Gamma(\operatorname{Ker}(c)) \subset \Gamma\left(H(M) \otimes \Sigma^{\ell}\right)
$$

denotes the $\ell$ th component of the twistor operator to $\theta$.

(b) A (non-trivial) element in the kernel of $\mathcal{P}_{\ell}$ is called $C R$ twistor spinor of weight $\ell$. The dimension $p_{\ell}$ of $\operatorname{Ker}\left(\mathcal{P}_{\ell}\right)$ denotes a $\mathrm{CR}$ invariant.

In the non-extremal cases, i.e., for $\ell \neq \pm m$, the twistor equation is overdetermined. In fact, similar as in [19], we suppose the existence of a twistor connection such that CR twistor spinors correspond to parallel sections in certain twistor bundles. This would imply that for $\ell \neq \pm m$ the CR invariants $p_{\ell}$ are numbers.

Example 7.3. Parallel spinors of weight $\ell \in\{-m,-m+2, \ldots, m-2, m\}$ are CR twistor spinors. For the spin case $(\ell=0)$ we discuss parallel spinors to any eigenvalue $\mu_{q}$ in [13]. They occur on pseudo-Einstein spin manifolds. In Section 12 we demonstrate the construction of closed CR manifolds admitting parallel spinors with $\ell=\mu_{q}=0$, i.e., CR twistor spinors.

Example 7.4. In [12] we describe pseudo-Hermitian Killing spinors for the case of a spin structure on $M$. These spinors are in the kernel of the twistor operator and realize a certain lower bound for the non-zero eigenvalues of the Kohn-Dirac operator $D_{\theta}$. In particular, we find CR twistor spinors of weight $\ell=0$ on the standard spheres $S^{2 m+1}$ of even CR dimension $m \geq 2$.

We also find Killing spinors in case that $\theta$ is related to some 3 -Sasakian structure on $M$. However, in this situation the CR dimension $m$ is odd and $\ell=0$ is impossible. Such Killing spinors are not CR twistor spinors.

\section{Vanishing theorems for harmonic spinors}

Let $\left(M^{2 m+1}, H(M), J\right), m \geq 1$, be strictly pseudoconvex with pseudo-Hermitian structure $\theta$ and Kohn-Dirac operator $D_{\theta}$ to some $\operatorname{spin}^{\mathbb{C}}$ structure of weight $\ell \in \mathbb{Z}$. The operator $D_{\theta}$ is formally self-adjoint and there exists a Schrödinger-Lichnerowicz-type formula (see [18]; cf. [12]). We use this formula to derive vanishing theorems for harmonic spinors.

Let $\nabla^{\text {tr }}$ denote the transversal part of the Webster-Tanaka spinorial derivative to a chosen $\operatorname{spin}^{\mathbb{C}}$ structure of weight $\ell \in \mathbb{Z}$. Then $\Delta^{\operatorname{tr}}=-\operatorname{tr}_{\theta}\left(\nabla^{\operatorname{tr}} \circ \nabla^{\operatorname{tr}}\right)$ denotes the spinor sub-Laplacian, and we have

$$
\Delta^{\operatorname{tr}}=\nabla_{10}^{*} \nabla_{10}+\nabla_{01}^{*} \nabla_{01},
$$


where $\nabla_{10}^{*}$ and $\nabla_{01}^{*}$ are the formal adjoint to $\nabla_{10}$ and $\nabla_{01}$, respectively. As in [12] we obtain with (5.1) the equation

$$
D_{\theta}^{2} \phi=\Delta^{\mathrm{tr}} \phi-\frac{\mathrm{i} \ell}{2(m+2)} \rho_{\theta} \phi+\frac{1}{4} \mathrm{scal}^{\mathrm{W}} \cdot \phi-\mathrm{d} \theta \nabla_{T}^{\Sigma} \phi
$$

for the square of the Kohn-Dirac operator. For the spinorial derivative in characteristic direction we compute

$$
\nabla_{T}^{\Sigma} \phi=\frac{\mathrm{i}}{4 m}\left(2 \nabla_{10}^{*} \nabla_{10}-2 \nabla_{01}^{*} \nabla_{01}+\mathrm{i} \rho_{\theta}-\frac{\ell \mathrm{scal}^{\mathrm{W}}}{2(m+2)}\right)(\phi) .
$$

This results in the Schrödinger-Lichnerowicz-type formula (cf. [12, 18])

$$
\begin{aligned}
D_{\theta}^{2}= & \left(\left(1-\frac{\Theta}{m}\right) \nabla_{10}^{*} \nabla_{10}+\left(1+\frac{\Theta}{m}\right) \nabla_{01}^{*} \nabla_{01}\right) \\
& -\frac{\mathrm{i}}{2}\left(\frac{\ell}{m+2}+\frac{\Theta}{m}\right) \rho_{\theta}+\left(1+\frac{\ell \Theta}{m(m+2)}\right) \frac{\mathrm{scal}^{\mathrm{W}}}{4} .
\end{aligned}
$$

The curvature part in (8.1) acts by Clifford multiplication as a self-adjoint operator on spinors. In case that this operator is positive definite, at each point of some closed manifold $M$, we immediately obtain vanishing results for harmonic spinors in the non-extremal bundles $\Sigma^{\mu_{q}}$ $\left(\mu_{q} \neq \pm m\right)$. We aim to specify the situation. Let us call the pseudo-Hermitian Ricci form $\rho_{\theta}$ positive (resp. negative) semidefinite if all eigenvalues are nonnegative (resp. nonpositive) on $M$. We can state our basic vanishing result as follows.

Proposition 8.1. Let $\theta$ be some pseudo-Hermitian structure on a closed CR manifold $M^{2 m+1}$, $m \geq 1$, with $\operatorname{spin}^{\mathbb{C}}$ structure of weight $\ell \in \mathbb{Z}$.

(a) A non-extremal bundle $\Sigma^{\mu_{q}}$ allows no harmonic spinors under the following conditions:

(1) $\mu_{q}=-\frac{m \ell}{m+2}$ and $\mathrm{scal}^{\mathrm{W}} \geq 0$ on $M$ with $\mathrm{scal}^{\mathrm{W}}(p)>0$ at some point $p \in M$.

(2) $\mu_{q}>-\frac{m \ell}{m+2}, \rho_{\theta}$ semidefinite on $M$ and $(m+2-\ell) \mathrm{scal}^{\mathrm{W}}>0$ at some point $p \in M$.

(3) $\mu_{q}<-\frac{m \ell}{m+2}, \rho_{\theta}$ semidefinite on $M$ and $(m+2+\ell) \mathrm{scal}^{\mathrm{W}}>0$ at some point $p \in M$.

(b) If $|\ell|>m+2$ and $\rho_{\theta} \not \equiv 0$ is negative semidefinite then any harmonic spinor is a section of the extremal bundles $\Sigma^{m} \oplus \Sigma^{-m}$.

(c) If $|\ell|<m+2$ and $\rho_{\theta} \not \equiv 0$ is positive semidefinite then any harmonic spinor is a section of the extremal bundles $\Sigma^{m} \oplus \Sigma^{-m}$.

Proof. Let $\phi=\phi_{\mu_{q}} \not \equiv 0$ be a spinor with $\Theta \phi=(m-2 q) \phi$. We put

$$
Q^{q}=-\frac{\mathrm{i}}{2}\left(\frac{\ell}{m+2}+\frac{\mu_{q}}{m}\right) \rho_{\theta}+\left(1+\frac{\ell \mu_{q}}{m(m+2)}\right) \frac{\mathrm{scal}^{\mathrm{W}}}{4}
$$

and

$$
A(\phi)=\int_{M}\left\langle Q^{q} \phi, \phi\right\rangle \operatorname{vol}_{\theta}
$$

The Schrödinger-Lichnerowicz-type formula (8.1) gives

$$
\left\|D_{\theta} \phi\right\|^{2}=\frac{2 q}{m}\left\|\nabla_{10} \phi\right\|^{2}+\frac{2(m-q)}{m}\left\|\nabla_{01} \phi\right\|^{2}+A(\phi) .
$$


If the eigenvalues of $\rho_{\theta}$ have no different signs, we have by Cauchy-Schwarz inequality

$$
\left|\left\langle\frac{\mathrm{i} \rho_{\theta}}{2} \cdot \phi, \phi\right\rangle\right| \leq \frac{\mathrm{scal}^{\mathrm{W}}}{4}|\phi|^{2} .
$$

Hence,

$$
A(\phi) \geq \frac{\left(m-\mu_{q}\right)(m+2-\ell)}{m(m+2)} \int_{M} \frac{\mathrm{scal}^{\mathrm{W}}}{4}|\phi|^{2} \operatorname{vol}_{\theta}
$$

for $m \ell+(m+2) \mu_{q} \geq 0$, and

$$
A(\phi) \geq \frac{\left(m+\mu_{q}\right)(m+2+\ell)}{m(m+2)} \int_{M} \frac{\mathrm{scal}^{\mathrm{W}}}{4}|\phi|^{2} \operatorname{vol}_{\theta}
$$

for $m \ell+(m+2) \mu_{q} \leq 0$.

We assume that $\mu_{q} \neq \pm m$ is non-extremal. Then $A(\phi)$ is obviously positive for the three cases of part (a) of the proposition. In particular, the right hand side of (8.3) is always positive. This shows that no harmonic spinors exist in these three cases.

If $|\ell|>m+2$ and $\rho_{\theta} \not \equiv 0$ is negative semidefinite then either condition (2) or (3) of part (a) is satisfied for any non-extremal $\Theta$-eigenvalue $\mu_{q} \neq \pm m$. If $|\ell|<m+2$ and $\rho_{\theta} \not \equiv 0$ is positive semidefinite, one of the three conditions of part (a) is satisfied for any non-extremal $\Theta$-eigenvalue $\mu_{q} \neq \pm m$.

Note that we have no vanishing results for harmonic spinors in the extremal bundles $\Sigma^{-m}$ or $\Sigma^{m}$. Such spinors are simply holomorphic or antiholomorphic, respectively. So far we also have no vanishing results for the canonical and anticanonical $\operatorname{spin}^{\mathbb{C}}$ structures when $\ell= \pm(m+2)$. However, there are vanishing results in these cases (see [21] and Section 10).

Example 8.2. Any pseudo-Einstein spin manifold $M$ (i.e., $\ell=0$ ) admits parallel spinors in the extremal bundles, no matter of the sign of the Webster scalar curvature (see [13]). For scal ${ }^{\mathrm{W}}>0$ these are the only harmonic spinors on closed $M$.

Let us consider the CR-covariant components $\mathcal{D}_{\ell}$ of the Kohn-Dirac operator. The formal adjoint of $\mathcal{D}_{\ell}, \ell \in\{-m,-m+2, \ldots, m-2, m\}$, is the restriction of $D_{\theta}$ to the image of $\mathcal{D}_{\ell}$, i.e.,

$$
\mathcal{D}_{\ell}^{*}=D_{\theta}: \operatorname{Im}\left(\mathcal{D}_{\ell}\right) \rightarrow \Gamma\left(\Sigma^{-\ell}\right) .
$$

Then the Schrödinger-Lichnerowicz-type formula (8.1) for $\mathcal{D}_{\ell}$ is expressed by

$$
\mathcal{D}_{\ell}^{*} \mathcal{D}_{\ell}=\frac{m+\ell}{m} \nabla_{10}^{*} \nabla_{10}+\frac{m-\ell}{m} \nabla_{01}^{*} \nabla_{01}+\frac{\mathrm{i} \ell \rho_{\theta}}{m(m+2)}+\left(1-\frac{\ell^{2}}{m(m+2)}\right) \frac{\mathrm{scal}^{\mathrm{W}}}{4} .
$$

Especially, for $\ell=0$, we have

$$
\mathcal{D}_{0}^{*} \mathcal{D}_{0}=\Delta^{\mathrm{tr}}+\frac{\mathrm{scal}^{\mathrm{W}}}{4}
$$

The latter formula looks like the classical Schrödinger-Lichnerowicz formula of Riemannian geometry. This immediately shows that in the spin case $h_{\frac{m}{2}}(\alpha)>0$ (i.e., a harmonic spinors of weight $\ell=0$ exists) poses an obstruction to the existence of any adapted pseudo-Hermitian structure on the CR manifold $(M, H(M), J)$ with positive Webster scalar scal ${ }^{\mathrm{W}}>0$. We give a more general version of this statement in terms of Kohn-Rossi cohomology in Corollary 10.4. Similarly, (8.4) implies that the CR invariants $\frac{h_{\frac{m+\ell}{2}}}{2}(\alpha)>0,|\ell|<m$, are obstructions to the positivity of the Ricci form $\rho_{\theta}>0$. 
Example 8.3. In Section 12 we construct closed CR manifolds over hyperKähler manifolds which admit harmonic spinors of weight $\ell=0$. Such CR manifolds admit no adapted pseudoHermitian structure $\theta$ of positive Webster scalar curvature.

Example 8.4. There exist compact quotients of the Heisenberg group, which are strictly pseudoconvex and spin with harmonic spinors of weight $\ell=0$ (see [20]).

\section{Harmonic theory for the Kohn-Rossi complex}

We briefly review here the Kohn-Rossi complex [8] over CR manifolds, twisted with some CR vector bundle $E$. With respect to a pseudo-Hermitian form we construct the Kohn Laplacian $\square_{E}$. Even though $\square_{E}$ is not an elliptic operator, there is a well behaving harmonic theory, similar to Hodge theory. In particular, Kohn-Rossi cohomology groups are finite and cohomology classes admit unique harmonic representatives over closed manifolds. This theory is due to J.J. Kohn (see $[4,7]$ ). Our exposition of the topic follows [21] by N. Tanaka.

Let $\left(M^{2 m+1}, H(M), J\right)$ be a closed manifold equipped with a strictly pseudoconvex CR structure of hypersurface type and $\mathrm{CR}$ dimension $m \geq 1$. With respect to the complex structure $J$ we have the decomposition $H(M) \otimes \mathbb{C}=T_{10} \oplus T_{01}$ of the Levi distribution. We define complex differential forms of degree $(p, q)$ on $H(M)$ by

$$
\Lambda^{p, q}(H(M)):=\Lambda^{p} T_{10}^{*} \otimes \Lambda^{q} T_{01}^{*}
$$

Then

$$
\Lambda^{r}(H(M)) \otimes \mathbb{C}=\bigoplus_{p+q=r} \Lambda^{p, q}(H(M))
$$

(Note that $(p, q)$-forms on $H(M)$ are not complex differentials form on $M$.)

We are interested in the bundles $\Lambda^{0, q}(H(M))=\Lambda^{q} T_{01}^{*}$ of $(0, q)$-forms. The corresponding spaces of smooth sections over $M$ are denoted by $\mathcal{C}^{q}(M), q=0, \ldots, m$. There exist tangential Cauchy-Riemann operators

$$
\bar{\partial}_{b}: \mathcal{C}^{q}(M) \rightarrow \mathcal{C}^{q+1}(M), \quad q \in\{0, \ldots, m\} .
$$

These differential operators are by construction CR invariants and the sequence

$$
0 \longrightarrow \mathcal{C}^{0}(M) \stackrel{\bar{\partial}_{b}}{\longrightarrow} \mathcal{C}^{1}(M) \stackrel{\bar{\partial}_{b}}{\longrightarrow} \cdots \stackrel{\bar{\partial}_{b}}{\longrightarrow} \mathcal{C}^{m-1}(M) \stackrel{\bar{\partial}_{b}}{\longrightarrow} \mathcal{C}^{m}(M) \longrightarrow 0
$$

is called Kohn-Rossi complex. Its cohomology groups are denoted by $H^{0, q}(M), q \geq 0$.

More generally, let us consider a complex vector bundle $E$ over $M$. We assume that $E$ is equipped with some Cauchy-Riemann operator

$$
\bar{\partial}_{E}: \Gamma(E) \rightarrow \Gamma\left(E \otimes T_{01}^{*}\right)
$$

i.e., $\bar{\partial}_{E}$ satisfies

$$
\begin{aligned}
& \bar{\partial}_{E}(f u)\left(X_{01}\right)=X_{01}(f) \cdot u+f \cdot\left(\bar{\partial}_{E} u\right)\left(X_{01}\right), \\
& \left(\bar{\partial}_{E} u\right)\left(\left[X_{01}, Y_{01}\right]\right)=\bar{\partial}_{E}\left(\bar{\partial}_{E} u\left(X_{01}\right)\right)\left(Y_{01}\right)-\bar{\partial}_{E}\left(\bar{\partial}_{E} u\left(Y_{01}\right)\right)\left(X_{01}\right)
\end{aligned}
$$

for any smooth $\mathbb{C}$-valued function $f$ and sections $X_{01}, Y_{01}$ in $T_{01}$. We call $\left(E, \bar{\partial}_{E}\right)$ a $C R$ vector bundle over $M$. Smooth sections $u$ of $E$ with $\bar{\partial}_{E} u=0$ are holomorphic sections (see [21]). 
Furthermore, for some CR vector bundle $E$ over $M$, we set $C^{q}(M, E)=\Lambda^{q} T_{01}^{*} \otimes E$ and $\mathcal{C}^{q}(M, E)=\Gamma\left(C^{q}(M, E)\right)$ for smooth sections. The holomorphic structure $\bar{\partial}_{E}$ on $E$ extends to Cauchy-Riemann operators

$$
\bar{\partial}_{E}: \mathcal{C}^{q}(M, E) \rightarrow \mathcal{C}^{q+1}(M, E)
$$

for any $q \in\{0, \ldots, m\}$. This is by construction a twisted complex $\left(\mathcal{C}^{q}(M, E), \bar{\partial}_{E}\right)$, and we denote the corresponding cohomology groups by $H^{q}(M, E), q \in\{0, \ldots, m\}$. For $E$ the trivial line bundle over $M$, these are the Kohn-Rossi cohomology groups $H^{0, q}(M)$ (see [21]).

Let us assume now that a pseudo-Hermitian form $\theta$ is given on $M$ and that the CR vector bundle $E \rightarrow M$ is equipped with a Hermitian inner product $\langle\cdot, \cdot\rangle_{E}$. In this setting we have a direct sum decomposition

$$
T(M) \otimes \mathbb{C}=T_{10} \oplus T_{01} \oplus \mathbb{R} T_{\theta},
$$

which gives rise to a unique identification of $C^{q}(M, E)$ with a subbundle of $\Lambda^{q}\left(T^{*}(M)\right) \otimes E$. And there exists a canonical connection $D: \Gamma(E) \rightarrow \Gamma\left(T^{*}(M) \otimes E\right)$ compatible with $\langle\cdot, \cdot\rangle_{E}$ and related to the Cauchy-Riemann operator by $D_{X_{01}} u=\bar{\partial}_{E} u\left(X_{01}\right), X_{01} \in T_{01}$, for any $u \in \Gamma(E)$. Together with the Webster-Tanaka connection $\nabla^{\mathrm{W}}$ we obtain covariant derivatives

$$
D: \Gamma\left(\Lambda^{q}(M) \otimes E\right) \rightarrow \Gamma\left(\Lambda^{q+1}(M) \otimes E\right), \quad q \in\{0, \ldots, m\},
$$

and with respect to a local frame $\left(E_{1}, \ldots, E_{m}\right)$ of $T_{10}$ the Cauchy-Riemann operators are given by

$$
\bar{\partial}_{E} u=\sum_{\alpha=1}^{m}{\overline{E_{\alpha}}}^{*} \wedge D_{\bar{E}_{\alpha}} u
$$

for $u \in \mathcal{C}^{q}(M, E)$.

Moreover, for any $q \in\{0, \ldots, m\}$, the vector bundle $C^{q}(M, E)$ is equipped with a Hermitian inner product, which gives rise via $\operatorname{vol}_{\theta}$ to an $L_{2}$-inner product on $\mathcal{C}^{q}(M, E)$. This allows the construction of a formally adjoint differential operator

$$
\bar{\partial}_{E}^{*}: \mathcal{C}^{q+1}(M, E) \rightarrow \mathcal{C}^{q}(M, E)
$$

to $\bar{\partial}_{E}$. With respect to a local frame $\left(E_{1}, \ldots, E_{m}\right)$ the operator $\bar{\partial}_{E}^{*}$ is given by

$$
\bar{\partial}_{E}^{*} u=-\sum_{\alpha=1}^{m} \iota \overline{E_{\alpha}} D_{E_{\alpha}} u
$$

for $u \in \mathcal{C}^{q+1}(M, E)$.

Finally, we can construct the Kohn Laplacian

$$
\square_{E}:=\bar{\partial}_{E}^{*} \bar{\partial}_{E}+\bar{\partial}_{E} \bar{\partial}_{E}^{*}: \mathcal{C}^{q}(M, E) \rightarrow \mathcal{C}^{q}(M, E), \quad q \in\{0, \ldots, m\},
$$

with respect to $\theta$ on $M$. This is a 2 nd order differential operator, which is formally self-adjoint with respect to $(\cdot, \cdot)_{L_{2}}$ on $\mathcal{C}^{q}(M, E)$. Due to results of Kohn the operator $\square_{E}$ is sub- and hypoelliptic. We put

$$
\mathcal{H}^{q}(M, E):=\left\{u \in \mathcal{C}^{q}(M, E) \mid \square_{E} u=0\right\}
$$

for the space of harmonic $(0, q)$-forms. Since $\square_{E}$ is formally self-adjoint the harmonic equation $\square_{E} u=0$ is equivalent to $\bar{\partial}_{E} u=\bar{\partial}_{E}^{*} u=0$ on $M$. It is known that $\mathcal{H}^{q}(M, E)$ is finite dimensional 
for any $q \in\{1, \ldots, m-1\}$. Moreover, every class in the Kohn-Rossi cohomology group $H^{q}(M, E)$ admits a unique harmonic representative, i.e.,

$$
H^{q}(M, E) \cong \mathcal{H}^{q}(M, E) .
$$

In particular, the Kohn-Rossi cohomology groups $H^{q}(M, E)$ are finite dimensional for $q \in$ $\{1, \ldots, m-1\}$. The groups $H^{0}(M, E)$ and $H^{m}(M, E)$ are infinite dimensional, in general. However, we still have $H^{0}(M, E) \cong \mathcal{H}^{0}(M, E)$ and $H^{m}(M, E) \cong \mathcal{H}^{m}(M, E)$ for any $m \geq 2$ (cf. [21]). In case $m=1$ and $M \subseteq \mathbb{C}^{2}$ is embedded as CR manifold $H^{0}(M, E) \cong \mathcal{H}^{0}(M, E)$ and $H^{1}(M, E) \cong \mathcal{H}^{1}(M, E)$ are certainly true as well.

\section{Vanishing theorems for twisted Kohn-Rossi cohomology}

The harmonic theory of the previous section fits well to our discussion of Kohn-Dirac operators and harmonic spinors. In fact, the square $D_{\theta}^{2}$ of the Kohn-Dirac operator has a natural interpretation as Kohn Laplacian $\square_{E}$ if we only make the appropriate choice for the CR line bundle $E$ (see [18]). This justifies the name for $D_{\theta}$ and gives rise via the Schrödinger-Lichnerowicz-type formula to vanishing results for twisted Kohn-Rossi cohomology (see in [21, Section II, § 7]; cf. [11]).

Let $\left(M^{2 m+1}, H(M), J\right)$ be a closed manifold equipped with strictly pseudoconvex CR structure of hypersurface type and $\mathrm{CR}$ dimension $m \geq 1$. We fix a pseudo-Hermitian form $\theta$ on $M$ with spin ${ }^{\mathbb{C}}$ structure of weight $\ell \in \mathbb{Z}$. The corresponding spinor bundle $\Sigma(H(M)) \rightarrow M$ decomposes into

$$
\Sigma(H(M))=\bigoplus_{q=0}^{m} \Sigma^{\mu_{q}}(H(M)) .
$$

The Kohn-Dirac operator is given by $D_{\theta}=D_{+}+D_{-}$. In particular, we have the spinorial complex (6.1) $\left(\Gamma\left(\Sigma^{\mu_{q}}\right), D_{+}\right)$with cohomology groups, which we denote by $S^{q}(M), q=0, \ldots, m$ (cf. the notion of spinorial cohomology in [15]).

Recall that the chosen $\operatorname{spin}^{\mathbb{C}}$ structure on $(M, \theta)$ is uniquely determined by some complex line bundle $E(\alpha) \rightarrow M, \alpha \in H^{2}(M, \mathbb{Z})$, which is a square root of $\mathcal{K} \otimes L, L=\mathcal{E}(\ell)$ the determinant bundle. Note that we can use the Webster-Tanaka connection $\nabla^{\mathrm{W}}$ to define a holomorphic structure on $E(\alpha)$ through $\bar{\partial}_{E(\alpha)} \eta\left(X_{01}\right):=\nabla_{X_{01}}^{\mathrm{W}} \eta, X_{01} \in T_{01}$, for $\eta \in \Gamma(E(\alpha))$.

Studying the spinor module $\Sigma$ with Clifford multiplication $c$ shows that the spinor bundle $\Sigma(H(M)) \rightarrow M$ is isomorphic to

$$
\bigoplus_{q=0}^{m} \Lambda^{q} T_{01}^{*} \otimes \Sigma^{\mu_{0}}(H(M)) .
$$

Moreover, the factor $\Sigma^{\mu_{0}}(H(M))$ is isomorphic to the line bundle $E(\alpha)$. In fact, we have $\Sigma^{\mu_{q}}(H(M)) \cong \Lambda^{q} T_{01}^{*} \otimes E(\alpha)$ of rank $\mathrm{rk}_{\mathbb{C}}=\left(\begin{array}{c}m \\ q\end{array}\right)$. Hence, the identifications

$$
\Gamma\left(\Sigma^{\mu_{q}}\right) \cong \mathcal{C}^{q}(M, E(\alpha)), \quad q=0, \ldots, m,
$$

with the chain groups of the Kohn-Rossi complex, twisted by $E(\alpha)$.

Examining the Clifford multiplication shows that the operator $\frac{1}{\sqrt{2}} D_{+}$corresponds via (10.1) to the Cauchy-Riemann operator $\bar{\partial}_{E(\alpha)}$. In particular, we have

$$
S^{q}(M) \cong H^{q}(M, E(\alpha)), \quad q \in\{0, \ldots, m\},
$$

for the cohomology groups of the spinorial complex. 
Moreover, the formal adjoint $\frac{1}{\sqrt{2}} D_{-}$corresponds to $\bar{\partial}_{E(\alpha)}^{*}$. Hence, via (10.1) we have

$$
D_{\theta}=\sqrt{2} \cdot\left(\bar{\partial}_{E(\alpha)}+\bar{\partial}_{E(\alpha)}^{*}\right)
$$

for the Kohn-Dirac operator and $D_{\theta}^{2}=2 \square_{E(\alpha)}$ for the square (cf. [18]). This shows that harmonic spinors with $\Theta$-eigenvalue $\mu_{q}=m-2 q, q=0, \ldots, m$, are in 1-to-1-correspondence with harmonic $(0, q)$-forms with values in $E(\alpha)$. In particular, with results of Section 9 we can interpret harmonic spinors on closed $M$ as representatives of twisted Kohn-Rossi cohomology classes.

Theorem 10.1. Let $\left(M^{2 m+1}, H(M), J\right), m \geq 2$, be a strictly pseudoconvex and closed $C R$ manifold with pseudo-Hermitian form $\theta$ and $\operatorname{spin}^{\mathbb{C}}$ structure, determined by $\alpha \in H^{2}(M, \mathbb{Z})$.

1. For the space of harmonic spinors to the eigenvalue $\mu_{q}, q=0, \ldots, m$, we have

$$
\mathcal{H}^{q}(\alpha) \cong \mathcal{H}^{q}(M, E(\alpha)) \cong H^{q}(M, E(\alpha)) \cong S^{q}(M) .
$$

2. The space $\mathcal{H}^{q}(\alpha)$ of harmonic spinors on $M$ is finite dimensional for any $q \in\{1, \ldots, m-1\}$.

Remark 10.2. The cohomology groups $H^{q}(M, E(\alpha)), q \in\{0, \ldots, m\}$, of the twisted KohnRossi complex are invariant objects of the underlying CR structure on $M$, whereas the construction of the spaces of harmonic spinors $\mathcal{H}^{q}(\alpha)$ and harmonic $(0, q)$-forms $\mathcal{H}^{q}(M, E(\alpha))$ depends on the pseudo-Hermitian structure $\theta$. It is only for $\mu_{q}=-\ell$ that we have seen in Section 7 that harmonic spinors in $\mathcal{H}^{\frac{m+\ell}{2}}(\alpha)$ are solutions of a $\mathrm{CR}$ invariant equation.

Theorem 10.1 shows now that elements in $\mathcal{H}^{q}(\alpha), q \in\{0, \ldots, m\}$, can be identified for different pseudo-Hermitian forms $\theta$ and $\tilde{\theta}$ via the corresponding Kohn-Rossi cohomology groups. In particular, all the dimensions $h_{q}(\alpha)$ of the spaces $\mathcal{H}^{q}(\alpha), q \in\{1, \ldots, m-1\}$, are CR invariant numbers.

Recall that a spin structure for the Levi distribution $H(M)$ on $M$ is given by some square root $E(\alpha)$ of the canonical bundle $\mathcal{K} \rightarrow M$. We denote the chosen square root by $\sqrt{\mathcal{K}}$. On the other hand, the canonical spin ${ }^{\mathbb{C}}$ structure on $M$ is given by the trivial line bundle $E(\alpha)=M \times \mathbb{C}$. In this case $\ell=m+2$. We have the following vanishing results for Kohn-Rossi cohomology.

Theorem 10.3. Let $\left(M^{2 m+1}, H(M), J\right), m \geq 2$, be a strictly pseudoconvex and closed CR manifold with pseudo-Hermitian form $\theta$ and $\operatorname{spin}^{\mathbb{C}}$ structure of weight $\ell$ determined by $\alpha \in$ $H^{2}(M, \mathbb{Z})$.

1. If $\rho_{\theta} \not \equiv 0$ is negative semidefinite, $|\ell|>m+2$ and $q \in\{1, \ldots, m-1\}$, then

$$
H^{q}(M, E(\alpha))=\{0\}
$$

for the qth ( $\alpha$-twisted) Kohn-Rossi cohomology group.

2. If $\rho_{\theta} \not \equiv 0$ is positive semidefinite, $|\ell|<m+2$ and $q \in\{1, \ldots, m-1\}$, then

$$
H^{q}(M, E(\alpha))=\{0\} .
$$

3. If $\rho_{\theta}>0$ is positive definite, $E(\alpha)=M \times \mathbb{C}$ and $q \in\{1, \ldots, m-1\}$, then

$$
H^{0, q}(M)=\{0\}
$$

for the qth Kohn-Rossi group.

4. If $\mathrm{scal}^{\mathrm{W}} \not \equiv 0$ is non-negative on a closed $C R$ spin manifold $M$ of even $C R$ dimension $m$, then

$$
H^{\frac{m}{2}}(M, \sqrt{\mathcal{K}})=\{0\} .
$$


Proof. Part (1) and (2) of Theorem 10.3 follow immediately from Proposition 8.1 via the identifications in Theorem 10.1. Part (3) is the statement of Proposition 7.4 on p. 62 in [21] for Kohn-Rossi cohomology. We reprove this result here.

The curvature term (8.2) decomposes into two summands as follows:

$$
\begin{aligned}
Q^{q} & =-\frac{\mathrm{i}}{2}\left(\frac{\ell}{m+2}+\frac{\mu_{q}}{m}\right) \rho_{\theta}+\left(1+\frac{\ell \mu_{q}}{m(m+2)}\right) \frac{\mathrm{scal}^{\mathrm{W}}}{4} \\
& =\left(1+\frac{\mu_{q}}{m}\right)\left(-\frac{\mathrm{i}}{2} \rho_{\theta}+\frac{\mathrm{scal}^{\mathrm{W}}}{4}\right)-\frac{\mathrm{i}(\ell-m-2)}{2(m+2)}\left(\rho_{\theta}-\frac{\mathrm{scal}^{\mathrm{W}} \cdot \mathrm{d} \theta}{4 m}\right) \\
& =: \frac{2(m-q)}{m} R_{*}+K .
\end{aligned}
$$

The second summand $K$ vanishes for $\ell=m+2$. In any case we have $\operatorname{tr}_{\theta} K=0$.

Via (10.1) Clifford multiplication on $(0, q)$-forms $u \in \Lambda^{q} T_{01}^{*}$ is given by

$$
X \cdot u=\sqrt{2} \cdot\left(X_{01}^{*} \wedge u-\iota_{X_{01}} u\right)
$$

for any $X \in H(M)$ (see, e.g., [16] and [18]). Then a short computations shows that $R_{*}=$ $-\frac{\mathrm{i}}{2} \rho_{\theta}+\frac{\mathrm{scal}^{\mathrm{W}}}{4}$ acts on $u \in C^{q}(M, E(\alpha))$ by

$$
\left(R_{*} u\right)\left(\bar{X}_{1}, \ldots, \bar{X}_{q}\right)=\sum_{\alpha=1}^{q} u\left(\bar{X}_{1}, \ldots, \rho_{\theta}\left(\bar{X}_{\alpha}\right), \ldots, \bar{X}_{q}\right)
$$

for any $\bar{X}_{1}, \ldots, \bar{X}_{q} \in \Lambda^{q} T_{01}^{*}$, i.e., $R_{*}$ is the Ricci operator on $C^{q}(M, E(\alpha))$.

On the other hand, the action of $K$ is induced by the curvature of the canonical connection on $E(\alpha)$ (which differs from the Webster-Tanaka connection, in general). Thus, $Q^{q}=$ $\frac{2(m-q)}{m} R_{*}+K$ is exactly the curvature term of the Weitzenböck formula in Proposition 5.1 on p. 47 of [21].

In particular, for the case of the canonical $\operatorname{spin}^{\mathbb{C}}$ structure $\alpha=0$, we have $\ell=m+2$ and $K=0$. If $\rho_{\theta}>0$ is positive definite on $M, R_{*}$ is positive definite as well. Application of the Weitzenböck formula for $\mathcal{C}^{q}(M, E(\alpha))$ shows that there are no harmonic forms.

Finally, in the spin case $\ell=0$ with even $m$, we have $Q^{\frac{m}{2}}=\frac{\mathrm{scal}^{\mathrm{W}}}{4}$ and (8.1) shows that there are no harmonic spinors. Hence, no harmonic forms in $\mathcal{C}^{\frac{m}{2}}(M, \sqrt{\mathcal{K}})$.

On the other hand, non-trivial Kohn-Rossi groups pose obstructions to positive Webster curvature on the underlying CR manifold. We put

$$
\hat{q}=\hat{q}(m, \ell):=\frac{m(m+\ell+2)}{2(m+2)}
$$

and highlight the following result, which resembles the classical obstruction for positive scalar curvature on Kähler manifolds (cf. [5, 14]).

Corollary 10.4. Let $\left(M^{2 m+1}, H(M), J\right), m \geq 2$, be a strictly pseudoconvex and closed CR manifold with $\operatorname{spin}^{\mathbb{C}}$ structure of weight $\ell \in \mathbb{Z}$ to $\alpha \in H^{2}(M, \mathbb{Z})$. If

$$
|\ell|<m+2, \quad \hat{q} \in \mathbb{Z} \quad \text { and } \quad H^{\hat{q}}(M, E(\alpha)) \neq\{0\},
$$

then $M$ admits no adapted pseudo-Hermitian structure $\theta$ of positive Webster scalar curvature $\mathrm{scal}^{\mathrm{W}}>0$.

Proof. For $\hat{q} \in \mathbb{Z}$, we have $\mu_{\hat{q}}=-\frac{m \ell}{m+2} \in \mathbb{Z}$ and $Q^{\hat{q}}=\left(1-\frac{\ell^{2}}{(m+2)^{2}}\right) \frac{\text { scal }^{\mathrm{W}}}{4}$. If $|\ell|<m+2$, then $\hat{q} \in\{1, \ldots, m-1\}$ and the functions $Q^{\hat{q}}$ and scal ${ }^{\mathrm{W}}$ have the same sign. The non-vanishing of $H^{\hat{q}}(M, E(\alpha))$ implies the existence of a harmonic spinor in $\Gamma\left(\Sigma^{\mu_{\hat{q}}}\right)$. This is impossible by (8.1) when scal $^{\mathrm{W}}>0$. 
Example 10.5. If $M$ is a closed and strictly pseudoconvex $\mathrm{CR}$ spin manifold of even $\mathrm{CR}$ dimension $m \geq 2$, then the cohomology group

$$
H^{\frac{m}{2}}(M, \sqrt{\mathcal{K}})
$$

poses an obstruction to scal ${ }^{\mathrm{W}}>0$. We give some concrete example of this case in Section 12 (cf. also Corollary 11.2).

Example 10.6. If $m=4$ and $\ell=-3$, then $\hat{q}=1$ and $H^{1}(M, E(\alpha))$ poses an obstruction to $\mathrm{scal}^{\mathrm{W}}>0$.

Example 10.7. Let $\left(M^{2 m+1}, \theta\right), m \geq 2$, be some pseudo-Einstein space with $\operatorname{Ric}^{\mathrm{W}}(T)=0$. Then scal ${ }^{\mathrm{W}}$ is constant on $M$. E.g., this happens when the Webster torsion $\tau$ is parallel, or when the characteristic vector $T_{\theta}$ is a transverse symmetry of the underlying CR structure (cf. Section 11).

We assume a $\operatorname{spin}^{\mathbb{C}}$ structure of weight $\ell$ and $\rho_{\theta} \not \equiv 0$. Then, either $\rho_{\theta}>0$ and, for $|\ell| \leq m+2$ and $1<q<m$, we have $H^{q}(M, E(\alpha))=\{0\}$ (by Theorem 10.3). E.g., Einstein-Sasakian manifolds of Riemannian signature give rise to such pseudo-Hermitian structures $\theta$ (see, e.g., [1] for the notion of Sasakian structures). In Section 12 we construct regular Einstein-Sasakian manifolds from Kähler geometry.

In the other case $\rho_{\theta}<0$ and $H^{q}(M, E(\alpha))=\{0\}$ for $|\ell|>m+2$ and any $1<q<m$. E.g., Einstein-Sasakian manifolds of Lorentzian signature fit to this situation.

\section{Cohomology of regular, torsion-free CR manifolds}

Let $\left(M^{2 m+1}, H(M), J\right), m \geq 2$, be strictly pseudoconvex with pseudo-Hermitian form $\theta$. We call the characteristic vector $T_{\theta}$ regular if all its integral curves are 1-dimensional submanifolds of $M$ and the corresponding leaf space $N$ is a smooth manifold of dimension $2 m$ with smooth projection $\pi: M \rightarrow N$. If, in addition, $\theta$ has vanishing Webster torsion $\tau=0$ then $T_{\theta}$ is an infinitesimal automorphism of the underlying CR structure and a Killing vector for the Webster metric $g^{\mathrm{W}}=g_{\theta}+\theta \circ \theta$ on $M$. Such a vector $T_{\theta}$ is called transverse symmetry. (Here we understand transverse to $H(M)$.) It is straightforward to see that in this case the pseudoHermitian structure $(H(M), J, \theta)$ projects to a Kähler structure on the leaf space $N$. Note that if $M$ is closed then $\pi: M \rightarrow N$ is a circle fiber bundle. In this case we call $\pi:(M, \theta) \rightarrow N$ a (regular, torsion-free) $C R$ circle bundle of complex dimension $m \geq 2$.

\subsection{Holomorphic cohomology and vanishing theorems}

Let us consider the underlying leaf space $N$ with Kähler metric $h$, complex structure $J$ and fundamental form $\omega$. As for any complex manifold, we have the $(p, q)$-forms $\Lambda^{p, q}(N)$ and the Cauchy-Riemann operators

$$
\bar{\partial}: \Gamma\left(\Lambda^{p, q}(N)\right) \rightarrow \Gamma\left(\Lambda^{p, q+1}(N)\right),
$$

which in turn give rise to the Dolbeault cohomology groups $H^{p, q}(N), p, q \geq 0$. In the following we are interested in the cohomology groups $H^{0, q}(N), q \geq 0$. In fact, more generally, let $E^{\prime} \rightarrow N$ be some holomorphic vector bundle and $\mathcal{O}\left(E^{\prime}\right)$ the sheaf of local holomorphic sections of $E^{\prime}$. Then we have the qth cohomology group $H^{q}\left(N, \mathcal{O}\left(E^{\prime}\right)\right)$ of the sheaf $\mathcal{O}\left(E^{\prime}\right)$, which is, by Dolbeault's theorem, isomorphic to $H^{0, q}\left(N, E^{\prime}\right), q \geq 0$.

Now let $E^{\prime}$ be some complex line bundle over the Kähler manifold $N$. We assume that $E^{\prime}$ is a root of some power of the anticanonical line bundle $\mathcal{K}^{-1} \rightarrow N$. This ensures that $E^{\prime}$ is equipped with a holomorphic structure and Hermitian inner product, both compatible with the 
Levi-Civita connection of the Kähler metric. The pullback $E=\pi^{*} E^{\prime}$ is a line bundle over $M$ with Hermitian inner product and the Webster-Tanaka connection induces some holomorphic structure on $E$ as well. Then any smooth section $u^{\prime} \in \Gamma\left(E^{\prime}\right)$ lifts to some smooth section $u=\pi^{*} u^{\prime}$ of $E \rightarrow M$. By construction, the Lie derivative $\mathfrak{L}_{T} u$ of the lift in characteristic direction vanishes identically on $M$. In fact, any smooth section $u \in \Gamma(E)$ with $\mathfrak{L}_{T} u=0$ is the pullback of some unique section $u^{\prime}$ in $E^{\prime} \rightarrow N$. We call such sections in $E \rightarrow M$ projectable. More generally, for $q \geq 0$, we have the subspaces

$$
\mathcal{C}_{(0)}^{q}(M, E) \subseteq \mathcal{C}^{q}(M, E)
$$

of projectable $(0, q)$-forms in the chain groups of the Kohn-Rossi complex with values in the line bundle $E$. These subgroups are naturally identified with the $(0, q)$-forms $\Gamma\left(\Lambda^{0, q} \otimes E^{\prime}\right)$ with values in $E^{\prime}$ over the Kähler manifold $N$.

Since the Webster torsion $\tau_{\theta}$ vanishes on $M$, the holomorphic structures on $E^{\prime}$ and on its pullback $E$ are compatible: $\bar{\partial}_{E} \pi^{*} v^{\prime}=\pi^{*} \bar{\partial}_{E^{\prime}} v^{\prime}$ for any $(0, q)$-form $v^{\prime}$ on $N$. Also $\bar{\partial}_{E}^{*} \pi^{*} v^{\prime}=\pi^{*} \bar{\partial}_{E^{\prime}}^{*} v^{\prime}$ is true. Now let $\gamma \in H^{0, q}\left(N, E^{\prime}\right)$ be a class in the $q$ th Dolbeault group. By classical Hodge theory $\gamma$ is uniquely represented by some harmonic $(0, q)$-form $u^{\prime}$ with values in $E^{\prime}$. In general, the lift $u=\pi^{*} u^{\prime}$ of some non-trivial harmonic $u^{\prime}$ is a non-trivial element of $\mathcal{C}_{(0)}^{q}(M, E)$, which is harmonic with respect to the Kohn Laplacian $\square_{E}$. Thus, we have an inclusion

$$
\pi^{*}: \mathcal{H}^{q}\left(N, E^{\prime}\right) \hookrightarrow \mathcal{H}^{q}(M, E)
$$

of spaces of harmonic forms. This again gives rise to a natural inclusion

$$
\pi^{*}: H^{q}\left(N, \mathcal{O}\left(E^{\prime}\right)\right) \hookrightarrow H^{q}(M, E)
$$

of the holomorphic cohomology group $H^{q}\left(N, \mathcal{O}\left(E^{\prime}\right)\right)$ into the Kohn-Rossi group $H^{q}(M, E)$ for any $q \geq 0$. We denote the image of this inclusion by $H_{(0)}^{q}(M, E)$. By construction, classes in $H_{(0)}^{q}(M, E)$ are represented by projectable harmonic $(0, q)$-forms on $M$, i.e., by elements of $\mathcal{H}_{(0)}^{q}(M, E)$.

Let us assume now that the given holomorphic line bundle $E^{\prime} \rightarrow N$ is a square root of $\mathcal{K}^{\prime} \otimes L^{\prime}$, where $L^{\prime} \rightarrow N$ is a line bundle of weight $\ell \in \mathbb{Z}$, i.e., $L^{\prime m+2}$ is the $\ell$ th power of the anticanonical bundle $\mathcal{K}^{\prime-1}$ over $N$. Then $E^{\prime}$ determines a $\operatorname{spin}^{\mathbb{C}}$ structure on $N$ with determinant bundle $L^{\prime}$ of weight $\ell$. This lifts to a $\operatorname{spin}^{\mathbb{C}}$ structure on $(M, \theta)$ with determinant bundle $L=\pi^{*} L^{\prime}$ of weight $\ell$. Of course, the corresponding spinor bundle $\Sigma^{\prime} \rightarrow N$ pulls back to the spinor bundle $\Sigma$ over $(M, \theta)$ and harmonic spinors in $\Sigma^{\prime}$ lift to harmonic spinors in $\Sigma \rightarrow M$.

Theorem 11.1. Let $\pi:(M, \theta) \rightarrow N$ be some $C R$ circle bundle of complex dimension $m \geq 2$ with spin $^{\mathbb{C}}$ structure of weight $|\ell|<m+2$ (determined by some line bundle $E=\pi^{*} E^{\prime} \rightarrow M$ ) and assume $\hat{q}=\frac{m(m+\ell+2)}{2(m+2)} \in \mathbb{Z}$.

(a) If the scalar curvature $\mathrm{scal}^{h} \not \equiv 0$ is non-negative on the Kähler manifold $N$ then there exist no harmonic spinors on $M$ to the $\Theta$-eigenvalue $\mu_{\hat{q}}=-\frac{m \ell}{m+2}$ and the Kohn-Rossi cohomology group $H^{\hat{q}}(M, E)=\{0\}$ is trivial.

(b) If the holomorphic cohomology group $H^{\hat{q}}\left(N, \mathcal{O}\left(E^{\prime}\right)\right) \neq\{0\}$ is non-trivial then the strictly pseudoconvex CR manifold $M$ admits no adapted pseudo-Hermitian structure of positive Webster scalar curvature.

Proof. Since $T_{\theta}$ is a transverse symmetry, we have $\iota_{T_{\theta}} R^{\mathrm{W}}=0$ for the Webster curvature operator. Hence, the pseudo-Hermitian Ricci form $\rho_{\theta}$ is the lift of the Ricci 2-form on $N$, and the Webster scalar curvature on $M$ is the lift of the Riemannian scalar curvature on $N$. 
If $\operatorname{scal}^{h} \not \equiv 0$ is non-negative on $N$, the curvature term $Q^{\hat{q}}=\left(1-\frac{\ell^{2}}{(m+2)^{2}}\right) \frac{\mathrm{scal}^{\mathrm{W}}}{4} \not \equiv 0$ is nonnegative as well. The Schrödinger-Lichnerowicz-type formula implies $H^{\hat{q}}(M, E)=\{0\}$. On the other hand, we have an inclusion of $H^{\hat{q}}\left(N, \mathcal{O}\left(E^{\prime}\right)\right)$ into $H^{\hat{q}}(M, E)$. Hence, if $H^{\hat{q}}\left(N, \mathcal{O}\left(E^{\prime}\right)\right)$ is non-trivial then $H^{\hat{q}}(M, E)$ as well. By Corollary 10.4, this obstructs the existence of some pseudo-Hermitian form with positive Webster scalar curvature.

In case of spin structures we have the following special result.

Corollary 11.2. Let $\pi:(M, \theta) \rightarrow N$ be some CR circle bundle of even complex dimension $m \geq 2$ with spin structure $\sqrt{\mathcal{K}^{\prime}} \rightarrow N$.

(a) If $\mathrm{scal}^{h} \not \equiv 0$ is non-negative on $N$, the Kohn-Rossi cohomology group

$$
H^{\frac{m}{2}}(M, \sqrt{\mathcal{K}})=\{0\}
$$

is trivial.

(b) If the holomorphic cohomology group $H^{\frac{m}{2}}\left(N, \mathcal{O}\left(\sqrt{\mathcal{K}^{\prime}}\right)\right) \neq\{0\}$ is non-trivial then the strictly pseudoconvex CR manifold $M$ admits no adapted pseudo-Hermitian structure of positive Webster scalar curvature.

\subsection{Shifting cohomology}

Let $\left(M^{2 m+1}, \theta\right), m \geq 2$, be some closed pseudo-Hermitian manifold with vanishing Webster torsion $\tau_{\theta}=0$. We assume now that the characteristic vector $T$ of $\theta$ is induced from some free $\mathrm{U}(1)$-action on $M$. This implies that $T$ is regular with underlying Kähler manifold $N$. In fact, here $\pi:(M, \theta) \rightarrow N$ is a smooth principal $\mathrm{U}(1)$-bundle and $\theta$ is a connection form. We call $\pi:(M, \theta) \rightarrow N$ a $C R$ principal $\mathrm{U}(1)$-bundle. The corresponding holomorphic line bundle is denoted by $F^{\prime} \rightarrow N$ and $F=\pi^{*} F^{\prime}$ is its pullback to $M$.

Let $\mathcal{C}^{q}(M)$ denote the chain groups of the Kohn-Rossi complex on $M$. In [21] the differential operator

$$
\begin{aligned}
\mathcal{N}: \mathcal{C}^{q}(M) & \rightarrow \mathcal{C}^{q}(M), \quad q \geq 0, \\
u & \mapsto \mathrm{i} \nabla_{T}^{\mathrm{W}} u,
\end{aligned}
$$

is introduced. Let us denote by

$$
\mathcal{C}_{(\lambda)}^{q}(M):=\left\{u \in \mathcal{C}^{q}(M): \mathcal{N} u=\lambda u\right\}
$$

the corresponding $\lambda$-eigenspace. Since the Webster torsion $\tau_{\theta}=0$ vanishes, we have in general $\nabla_{T}^{\mathrm{W}} u=\mathfrak{L}_{T} u$ for the Lie derivative of any chain $u \in \mathcal{C}^{q}(M)$. This shows that $\mathcal{C}_{(0)}^{q}(M)$ are exactly the projectable $(0, q)$-forms on $M$.

The operator $\mathcal{N}$ is self-adjoint and commutes with $\bar{\partial}$ and $\bar{\partial}^{*}$. Hence, $\mathcal{N}$ acts on the harmonic spaces $\mathcal{H}^{0, q}(M), q \geq 0$, as well. All eigenvalues $\lambda$ are real. In fact, since $\mathcal{H}^{0, q}(M)$ is finite dimensional for $0<q<m$, we have finitely many real eigenvalues $\lambda$ and a direct sum decomposition

$$
\mathcal{H}^{0, q}(M)=\bigoplus_{\lambda} \mathcal{H}_{(\lambda)}^{0, q}(M)
$$

which implies for the Kohn-Rossi groups:

$$
H^{0, q}(M)=\bigoplus_{\lambda} H_{(\lambda)}^{0, q}(M), \quad 1 \leq q \leq m-1 .
$$


In [21] it is shown that for any integers $q, s \in \mathbb{Z}$ the cohomology groups

$$
H_{(s)}^{0, q}(M) \cong H^{q}\left(N, \mathcal{O}\left(F^{s}\right)\right)
$$

are naturally identified. Here $F^{\prime s}$ denotes the $s$ th power of the line bundle $F^{\prime} \rightarrow N$, which is associated to the given CR principal $\mathrm{U}(1)$-bundle $\pi:(M, \theta) \rightarrow N$.

On the other hand, let $F=\pi^{*} F^{\prime} \rightarrow M$ denote the pullback of $F^{\prime}$. Via the projection $\pi: M \rightarrow N$ the holomorphic cohomology group $H^{q}\left(N, \mathcal{O}\left(F^{\prime s}\right)\right)$ is identified with the subgroup $H_{(0)}^{q}\left(M, F^{s}\right) \subseteq H^{q}\left(M, F^{s}\right)$ of $F^{s}$-twisted Kohn-Rossi cohomology. This gives rise to a natural identification

$$
H_{(s)}^{0, q}(M) \cong H_{(0)}^{q}\left(M, F^{s}\right),
$$

where the classes in $H_{(0)}^{q}\left(M, F^{s}\right)$ are uniquely represented by projectable harmonic $(0, q)$-forms with values in $F^{s}$. We call (11.1) a shift in Kohn-Rossi cohomology over the CR principal $\mathrm{U}(1)$-bundle $\pi:(M, \theta) \rightarrow N$.

We apply (11.1) to our situation of $\operatorname{spin}^{\mathbb{C}}$ structures. For this we assume that the pullback $F=\pi^{*} F^{\prime} \rightarrow M$ of the line bundle $F^{\prime} \rightarrow N$ associated to $\pi: M \rightarrow N$ has weight $f \in \mathbb{Z}$. Then $E:=F^{s}=\mathcal{E}(s f)$ determines a $\operatorname{spin}^{\mathbb{C}}$ structure of weight $\ell=2 s f+m+2$ on $(M, \theta)$.

Theorem 11.3. Let $\pi:\left(M^{2 m+1}, \theta\right) \rightarrow N, m \geq 2$, be some CR principal $\mathrm{U}(1)$-bundle of weight $f \in \mathbb{Z}$ and $E:=\mathcal{E}(s f), s \in \mathbb{Z}$. Then

1. $H_{(0)}^{q}(M, E)=\{0\}$ for $q<m$ and $s>0$.

2. If $\frac{m s f}{m+2} \in\{1-m, \ldots,-1\}$ and $\mathrm{scal}^{h}>0$ then $H_{(s)}^{0, \hat{q}}(M)=\{0\}$ for $\hat{q}=m\left(1+\frac{s f}{m+2}\right)$.

Proof. (1) Comparing the Kohn Laplacian $\square=\bar{\partial}^{*} \bar{\partial}+\bar{\partial} \bar{\partial}^{*}$ with $\bar{\square}=\partial^{*} \partial+\partial \partial^{*}$ gives $\bar{\square}+(m-$ $q) \mathcal{N}=\square$ (see [21]). Hence, the subgroups $H_{(0)}^{q}(M, E) \cong H_{(s)}^{0, q}(M), q<m$, are all trivial for eigenvalues $s>0$.

(2) This result follows directly from Theorem 11.1 and the shift (11.1).

Example 11.4. Let us assume that the CR dimension $m \geq 2$ is even and the weight $f$ of $F \rightarrow N$ is a positive factor of $\frac{m}{2}+1$, i.e., $s:=-\frac{m+2}{2 f} \in \mathbb{Z}$. Then $E^{2}=F^{2 s}=\mathcal{K}$, i.e., $F^{s} \rightarrow M$ defines a spin structure on the CR manifold $M$. Hence, if the scalar curvature scal ${ }^{h}$ of the underlying Kähler manifold is positive, the Kohn-Rossi subgroup $H_{(s)}^{0, \frac{m}{2}}(M) \cong H_{(0)}^{\frac{m}{2}}\left(M, F^{s}\right)$ is trivial.

In other words, in this situation the (untwisted) Kohn-Rossi subgroup

$$
H_{(s)}^{0, \frac{m}{2}}(M) \neq\{0\}
$$

to the negative $\mathcal{N}$-eigenvalue $s=-\frac{m+2}{2 f} \in \mathbb{Z}$ is an obstruction to positive Webster scalar curvature on $M$.

\section{Examples of CR principal U(1)-bundles}

We discuss some examples of CR principal U(1)-bundles with applications of vanishing theorems for harmonic spinors and Kohn-Rossi cohomology. The construction here is based on the underlying Kähler manifold.

For our construction, let $\left(N^{2 m}, \omega, J\right), m \geq 2$, be a closed Kähler manifold such that a nontrivial multiple $\alpha:=c[\omega], c \in \mathbb{R} \backslash\{0\}$, of the Kähler class $[\omega] \neq 0$ is integral, i.e., $\alpha \in H^{2}(N, \mathbb{Z})$. By Lefschetz's theorem on $(1,1)$-classes, there exists some Hermitian line bundle $\mathcal{L}^{\prime} \rightarrow N$ with first Chern class $c_{1}\left(\mathcal{L}^{\prime}\right)=\alpha$. Let $\pi: \mathcal{M} \rightarrow N$ be the corresponding principal U(1)-bundle to 
which $\mathcal{L}^{\prime} \rightarrow N$ is associated. By the $d d^{c}$-lemma, this bundle admits a connection form $A_{\omega}$ with curvature $\mathrm{d} A_{\omega}=\pi^{*} \omega$, the lift of the Kähler form. The lift of the complex structure $J$ to the horizontal distribution $H(\mathcal{M})$ of the connection $A_{\omega}$ gives rise to a CR structure $(H(\mathcal{M}), J)$ on $\mathcal{M}$, which is by construction strictly pseudoconvex of hypersurface type with CR dimension $m$.

In addition, we set $\theta:=2 A_{\omega}$. This is an adapted pseudo-Hermitian form, which has by construction vanishing torsion $\tau=0$, the characteristic vector $T$ is regular and $g_{\theta}=\frac{1}{2} \mathrm{~d} \theta(\cdot, J \cdot)$ is the lift of the Kähler metric to $H(\mathcal{M})$. Thus,

$$
(\mathcal{M}, H(\mathcal{M}), J)
$$

is a CR principal U(1)-bundle with pseudo-Hermitian form $\theta$ over the Kähler manifold $N$. Let us call $\pi: \mathcal{M} \rightarrow N$ the $C R \alpha$-bundle.

Example 12.1. Let $N^{2 m}$ be a closed Kähler-Einstein manifold of positive scalar curvature scal $^{h}>0$ and let $\pi: \mathcal{M} \rightarrow N$ be the principal U(1)-bundle to which the anticanonical bundle $\mathcal{K}^{\prime-1} \rightarrow N$ is associated. The Ricci 2 -form is given by $\rho=\frac{\mathrm{scal}^{h}}{2 m} \omega$ and the first Chern class is $c_{1}(N)=-c_{1}\left(\mathcal{K}^{\prime}\right)=\left[\frac{1}{2 \pi} \rho\right] \in H^{2}(N, \mathbb{Z})$. Hence, $\pi: \mathcal{M} \rightarrow N$ is the CR $c_{1}(N)$-bundle. The adapted pseudo-Hermitian form $\theta$ is some multiple of the Levi-Civita connection on $\mathcal{M}$, and the lift of the Ricci 2-form $\rho$ along $\pi: \mathcal{M} \rightarrow N$ is the pseudo-Hermitian Ricci form $\rho_{\theta}$ of $\theta$ on $\mathcal{M}$. This shows that $\rho_{\theta}$ is positive definite in this case.

Let $\mathcal{M}$ be given some $\operatorname{spin}^{\mathbb{C}}$ structure of weight $\ell \in \mathbb{Z}$, determined by some line bundle $E \rightarrow \mathcal{M}$. For $|\ell|<m+2$, Proposition 8.1 shows that any harmonic spinor on $\mathcal{M}$ is a section of the extremal bundles. Accordingly, Theorem 10.3 shows that, for $|\ell| \leq m+2$ and any $1 \leq q \leq m-1$, the $q$ th Kohn-Rossi group $H^{q}(\mathcal{M}, E)=\{0\}$ is trivial. In particular, if $N^{2 m}$ ( $m$ even) is spin, so is $\mathcal{M}$ and $H^{\frac{m}{2}}(\mathcal{M}, \sqrt{\mathcal{K}})=\{0\}$.

For example, the Hopf fibration $\pi: S^{2 m+1} \rightarrow \mathbb{C} P^{m}$ over the complex projective space is a $\mathrm{CR}$ principal U(1)-bundle of this kind. In fact, the associated line bundle to $\pi$ is $\mathcal{K}^{\prime-1}$, and $S^{2 m+1}$ is equipped with the standard $C R$ structure. Hence, for any $\operatorname{spin}^{\mathbb{C}}$ structure of weight $|\ell| \leq m+2$ and $0<q<m$, we have $H^{q}\left(S^{2 m+1}, E\right)=\{0\}$ (cf. [21]). (Note that $\mathbb{C} P^{m}$ is spin only for $m$ odd. However, the standard CR manifold $S^{2 m+1}$ is spin for any $m$.)

Example 12.2. Let $N^{2 m}, m \geq 2$ and even, be some closed manifold with hyperKähler metric $h$, i.e., $h$ is Ricci-flat and its holonomy group is contained in $\operatorname{Sp}\left(\frac{m}{2}\right)$. In particular, $N$ is spin and admits some parallel spinor $\phi_{0}$ in $\Sigma^{0}(N)$, i.e., $H^{\frac{m}{2}}\left(N, \mathcal{O}\left(\sqrt{\mathcal{K}^{\prime}}\right)\right) \neq\{0\}$.

We assume now that $h$ is a Hodge metric, i.e., the corresponding Kähler class $[\omega] \in H^{2}(N, \mathbb{Z})$ is integral. E.g., any projective K3 surface admits some integral Kähler class [ $\omega]$. Then the CR [ $\omega]$-bundle $\pi: \mathcal{M} \rightarrow N$ exists. Furthermore, the pullback of the spin structure on $N$ gives rise to some spin structure $\sqrt{\mathcal{K}} \rightarrow \mathcal{M}$ and the lift $\psi_{0}:=\pi^{*} \phi_{0}$ is a parallel section of $\Sigma^{0}(\mathcal{M}(H))$ with respect to the spinorial Webster-Tanaka connection $\nabla^{\Sigma}$. In fact, the basic holonomy group of the adapted pseudo-Hermitian structure $\theta$ on $\mathcal{M}$ is contained in $\operatorname{Sp}\left(\frac{m}{2}\right)$ (cf. [13]). In particular, $\psi_{0}$ is some harmonic spinor in $\Sigma^{0}(\mathcal{M}(H))$ and the Kohn-Rossi group $H^{\frac{m}{2}}(\mathcal{M}, \sqrt{\mathcal{K}}) \neq\{0\}$ is non-trivial. Accordingly, Corollary 10.4 states that the $\mathrm{CR}$ manifold $\mathcal{M}$ admits no pseudoHermitian structure of positive Webster scalar curvature. Similarly, we can apply Corollary 11.2, since $H^{\frac{m}{2}}\left(N, \mathcal{O}\left(\sqrt{\mathcal{K}^{\prime}}\right)\right) \neq\{0\}$.

Observe that $\psi_{0}$ is in the kernel of the twistor operator $\mathcal{P}_{0}$ as well, i.e., $\psi_{0}$ is an example for a CR twistor spinor of weight $\ell=0$ on some closed, strictly pseudoconvex CR manifold.

Finally, note that in the spin case there is a theory of Kählerian twistor spinors on the underlying $N$ (see $[17,19]$ ). The lift of such twistor spinors gives rise to projectable spinors in the kernel of twistor operators on $(M, \theta)$. For the case $\mu_{q}=\ell=0$, this gives rise to CR twistor spinors. However, Kählerian twistor spinors on $N$ with $\mu_{q}=0$ are necessarily parallel. Hence, as in our example, the corresponding CR twistor spinors on $M$ are parallel as well. Apart from 
the standard CR sphere, we do not know further examples (of non-extremal weight) on closed CR manifolds.

\section{Acknowledgements}

I would like to thank the referees for their helpful comments.

\section{References}

[1] Blair D.E., Contact manifolds in Riemannian geometry, Lecture Notes in Math., Vol. 509, Springer-Verlag, Berlin - New York, 1976.

[2] Čap A., Gover A.R., CR-tractors and the Fefferman space, Indiana Univ. Math. J. 57 (2008), 2519-2570 arXiv:math.DG/0611938.

[3] Case J.S., Yang P., A Paneitz-type operator for CR pluriharmonic functions, Bull. Inst. Math. Acad. Sin. (N.S.) 8 (2013), 285-322, arXiv:1309.2528.

[4] Folland G.B., Kohn J.J., The Neumann problem for the Cauchy-Riemann complex, Annals of Mathematics Studies, Vol. 75, Princeton University Press, Princeton, N.J., University of Tokyo Press, Tokyo, 1972.

[5] Hitchin N., Harmonic spinors, Adv. Math. 14 (1974), 1-55.

[6] Jerison D., Lee J.M., The Yamabe problem on CR manifolds, J. Differential Geom. 25 (1987), $167-197$.

[7] Kohn J.J., Boundaries of complex manifolds, in Proc. Conf. Complex Analysis (Minneapolis, 1964), Springer, Berlin, 1965, 81-94.

[8] Kohn J.J., Rossi H., On the extension of holomorphic functions from the boundary of a complex manifold, Ann. of Math. 81 (1965), 451-472.

[9] Kordyukov Y.A., Vanishing theorem for transverse Dirac operators on Riemannian foliations, Ann. Global Anal. Geom. 34 (2008), 195-211, arXiv:0708.1698.

[10] Lawson Jr. H.B., Michelsohn M.L., Spin geometry, Princeton Mathematical Series, Vol. 38, Princeton University Press, Princeton, NJ, 1989.

[11] Lee J.M., Pseudo-Einstein structures on CR manifolds, Amer. J. Math. 110 (1988), 157-178.

[12] Leitner F., The first eigenvalue of the Kohn-Dirac operator on CR manifolds, Differential Geom. Appl. 61 (2018), 97-132.

[13] Leitner F., Parallel spinors and basic holonomy in pseudo-Hermitian geometry, Ann. Global Anal. Geom. 55 (2019), 181-196.

[14] Lichnerowicz A., Spineurs harmoniques, C. R. Acad. Sci. Paris 257 (1963), 7-9.

[15] Michelsohn M.L., Clifford and spinor cohomology of Kähler manifolds, Amer. J. Math. 102 (1980), 10831146.

[16] Morgan J.W., The Seiberg-Witten equations and applications to the topology of smooth four-manifolds, Mathematical Notes, Vol. 44, Princeton University Press, Princeton, NJ, 1996.

[17] Moroianu A., On Kirchberg's inequality for compact Kähler manifolds of even complex dimension, Ann. Global Anal. Geom. 15 (1997), 235-242.

[18] Petit R., Spin ${ }^{c}$-structures and Dirac operators on contact manifolds, Differential Geom. Appl. 22 (2005), 229-252.

[19] Pilca M., Kählerian twistor spinors, Math. Z. 268 (2011), 223-255, arXiv:0812.3315.

[20] Stadtmüller C., Horizontal Dirac operators in CR geometry, Ph.D. Thesis, Humboldt University Berlin, 2017, available at https://edoc.hu-berlin.de/handle/18452/18801.

[21] Tanaka N., A differential geometric study on strongly pseudo-convex manifolds, Lectures in Mathematics, Vol. 9, Kinokuniya Book-Store Co., Ltd., Tokyo, 1975.

[22] Webster S.M., Pseudo-Hermitian structures on a real hypersurface, J. Differential Geometry 13 (1978), 25-41. 